\title{
A normal abundance of faint satellites in the fossil group NGC $6482^{\star, \star \star}$
}

\author{
S. Lieder ${ }^{1,2}$, S. Mieske ${ }^{2}$, R. Sánchez-Janssen²,3 ${ }^{2}$ M. Hilker ${ }^{4}$, T. Lisker ${ }^{1}$, and M. Tanaka ${ }^{5}$ \\ ${ }^{1}$ Astronomisches Rechen-Institut, Zentrum für Astronomie der Universität Heidelberg, Mönchhofstraße 12-14, 69120 Heidelberg, \\ Germany \\ e-mail: slieder@ari.uni-heidelberg.de \\ 2 European Southern Observatory, Av. Alonso de Córdova 3107, Casilla 19001, Vitacura, Santiago, Chile \\ 3 NRC Herzberg Institute of Astrophysics, 5071 West Saanich Road, Victoria BC V9E 2E7, Canada \\ ${ }^{4}$ European Southern Observatory, Karl-Schwarzschild-Strasse 2, 85748 Garching bei München, Germany \\ 5 National Astronomical Observatory of Japan, 2-21-1 Osawa, Mitaka, 181-8588 Tokyo, Japan
}

Received 13 February 2013 / Accepted 3 September 2013

\begin{abstract}
A fossil group is considered the end product in a galaxy group's evolution. It is a massive central galaxy that dominates the luminosity budget of the group, and is the outcome of efficient merging between intermediate-luminosity members. Little is known, however, about the faint satellite systems of fossil groups. Here we present a Subaru/Suprime-Cam wide-field, deep imaging study in the $B$ - and $R$-bands of the nearest fossil group NGC $6482\left(M_{\mathrm{tot}} \sim 4 \times 10^{12} M_{\odot}\right)$, covering the virial radius out to $310 \mathrm{kpc}$. We performed detailed completeness estimations and selected group member candidates by a combination of automated object detection and visual inspection. A fiducial sample of 48 member candidates down to $M_{R} \sim-10.5$ mag is detected, making this study the deepest of a fossil group to now. We investigate the photometric scaling relations, the color-magnitude relation, and the luminosity function of our galaxy sample. We find evidence of recent and ongoing merger events among bright group galaxies. The color-magnitude relation is comparable to that of nearby galaxy clusters, and it exhibits significant scatter at the faintest luminosities. The completeness-corrected luminosity function is dominated by early-type dwarfs and is characterized by a faint end slope $\alpha=-1.32 \pm 0.05$. We conclude that the NGC 6482 fossil group shows photometric properties consistent with those of regular galaxy clusters and groups, including a normal abundance of faint satellites.
\end{abstract}

Key words. galaxies: evolution - galaxies: dwarf - galaxies: groups: general - galaxies: clusters: general - galaxies: photometry

\section{Introduction}

The study of so-called "fossil groups" (FGs) began about two decades ago. Ponman et al. (1994) found the first of these systems: X-ray luminous galaxy groups characterized by their dominant bright central elliptical galaxy resulting in high massto-light ratios. The formal definition by Jones et al. (2003), generally adopted by the community, is the following: (1) ensure that there is a dominant galaxy in the group by adopting an $R$-band magnitude gap $\Delta m_{12}$ of at least two magnitudes between the two most luminous galaxies. They restrict that criterion to galaxies within half of the group's projected virial radius $r_{\text {vir }}$ to ensure that $L^{*}$ galaxies have had enough time to merge owing to dynamical friction, since the merging timescale within $0.5 r_{\text {vir }}$ for these systems is shorter than a Hubble time (Zabludoff \& Mulchaey 1998). (2) Exclude "normal" elliptical galaxies that are not located in the center of the group by finding a hot gas halo that surrounds the galaxy (typical of central galaxies). This is achieved by a minimum X-ray luminosity of $L_{\mathrm{X}, \mathrm{bol}}=10^{42} h_{50}^{-2} \mathrm{erg} / \mathrm{s}$.

\footnotetext{
* Appendix A is available in electronic form at http: //www . aanda.org

$\star \star$ The reduced data are only available at the CDS via anonymous ftp to cdsarc.u-strasbg.fr $(130.79 .128 .5)$ or via

http://cdsarc.u-strasbg.fr/viz-bin/qcat?]/A+A/559/A76
}

Ponman et al. (1994) interpreted their observations as witnessing the final stage in a group's evolution: an ancient stellar population in which most of the group's bright galaxies have merged into one luminous galaxy. They therefore termed their finding a "fossil" group. This evolutionary scenario seems a plausible one for isolated parts in our Universe where galaxy groups can evolve undisturbed. Another FG formation scenario has been suggested by Mulchaey \& Zabludoff (1999). Here, FGs could merely be "failed" groups in which the majority of the baryonic mass was accidentally placed in a single dark matter halo, leading to the dominant central object. Investigations of FGs do not favor this scenario since several studies have revealed that fossil brightest group galaxies (BGGs) exhibit disky isophotes (Khosroshahi et al. 2006). According to Bender (1988) and Khochfar \& Burkert (2005), it is considered that gas-rich mergers cause this isophotal behavior. This is also supported by Aguerri et al. (2011) and Méndez-Abreu et al. (2012), who find that the Sérsic index of BGGs in FGs is significantly smaller than that in central galaxies of clusters. According to Hopkins et al. (2009), small Sérsic $n$ stem from gas-rich mergers.

Investigations based on the criteria by Jones et al. (2003) have generally led to the conclusion that FGs formed early and have not experienced any major merger event for several Gyr (Sanderson et al. 2003; Khosroshahi et al. 2004). According to simulations, they accreted the majority of their mass at high 
redshifts (e.g., $50 \%$ at $z>1$; D'Onghia et al. 2005; Dariush et al. 2007; Díaz-Giménez et al. 2008). Fossil groups would therefore constitute the top end of the hierarchical evolution of galaxies on group scales.

Some more recent studies suggest that FGs may only be a transient phase in a group's evolution (von Benda-Beckmann et al. 2008; Dariush et al. 2010; Cui et al. 2011). Dariush et al. (2010) argue that most of the early formed systems are not in a "fossil phase" at $z=0$, but were so at some earlier point during their evolution. It is also clear that the observational criteria for FG classification are to some extent arbitrary, and slight changes to, say, $\Delta m_{12}$ will change the fraction of environments classified as fossil. Milosavljevi et al. (2006) show that there is a smooth distribution of the luminosity gap among 730 SDSS clusters, in line with the idea that the observational definition of a fossil group does not necessarily highlight a marked change in underlying formation histories. Nevertheless, it is clear that fossil groups mark an extreme environment (in the tail of a smooth distribution), which is only expected in a few per cent of massive dark matter halos (Milosavljevi et al. 2006).

Here we are interested in the properties of the faint galaxy population in such an extreme environment. Early results suggested that FGs also lacked faint satellites (Jones et al. 2000) that provide potentially interesting constraints on the so-called "substructure crisis/missing satellite problem” of $\Lambda \mathrm{CDM}$ (D'Onghia $\&$ Lake 2004). Recently, Mendes de Oliveira et al. (2009) has reanalyzed the FG used by D'Onghia \& Lake (2004) and found a steeper faint end slope of -1.6 - comparable to clusters (e.g., Coma cluster: $\alpha=-1.4$, Secker et al. 1997). Other recent studies of FGs reveal shallower faint end slopes of $\alpha=-1.2$ when determined out to $\sim r_{\text {vir }}$ (Cypriano et al. 2006; Proctor et al. 2011; Eigenthaler \& Zeilinger 2012), whereas the restriction to $0.5 r_{\text {vir }}$ even reveals declining faint ends, i.e., $\alpha>-1.0$ (Mendes de Oliveira et al. 2006; Aguerri et al. 2011; Proctor et al. 2011).

However, none of these studies reaches magnitudes fainter than $M_{R} \sim-17 \mathrm{mag}^{1}$, barely scratching the dwarf regime. Therefore they cannot provide meaningful constraints on the asymptotic faint end slope of the galaxy luminosity function. To investigate this faint galaxy population of an FG, we provide here a photometric analysis of the NGC 6482 group down to $M_{R} \sim-10.5 \mathrm{mag}$ - to our knowledge the deepest FG study yet.

\section{Data}

\subsection{NGC 6482}

NGC 6482 is the nearest known FG ( $z=0.0131$; Smith et al. 2000), so it is well suited for a ground-based study of its dwarf galaxy population. If adopting the cosmological parameters $H_{0}=70.0 \mathrm{~km} \mathrm{~s}^{-1} \mathrm{Mpc}^{-1}, \Omega_{\mathrm{M}}=0.3$, and $\Omega_{\Lambda}=0.7$, NGC 6482's distance is $m-M=33.7 \mathrm{mag}(d=55.7 \mathrm{Mpc})$, resulting in a physical scale of $0.263 \mathrm{kpc} \mathrm{arcsec}^{-1}$. We use these numbers throughout the paper.

We anticipate here that we measure $M_{R}=-22.7 \mathrm{mag}$ for the BGG and for the second ranked galaxy $M_{R}=-20.5 \mathrm{mag}$. Together with the X-ray luminosity of $L_{\mathrm{X}}=1.0 \times 10^{42} h_{70}^{-2} \mathrm{erg} \mathrm{s}^{-1}$ (Böhringer et al. 2000) NGC 6482 meets the fossil definition by Jones et al. (2003). Chandra observations imply a virial radius of $310 \mathrm{kpc}$, a hot gas mass fraction of $f_{\text {gas }}=0.16$, and a total mass of $M_{200} \approx 4 \times 10^{12} M_{\odot}$, with an $R$-band mass-to-light ratio

\footnotetext{
1 Deeper observations of FGs exist with the HST ACS, but their much more constrained spatial coverage makes them inappropriate for the study of widely distributed dwarf galaxies.
}

Table 1. $R$-band observation time line from 5 June 2008 .

\begin{tabular}{lcccc}
\hline \hline Image type & Exp. time & Airmass & HST & Note \\
\hline Science & $60 \mathrm{~s}$ & 1.024 & $2: 07$ & \\
Science & $60 \mathrm{~s}$ & 1.026 & $2: 09$ & \\
Science & $540 \mathrm{~s}$ & 1.036 & $2: 11$ & $a$ \\
Science & $540 \mathrm{~s}$ & 1.048 & $2: 21$ & \\
Science & $540 \mathrm{~s}$ & 1.062 & $2: 31$ & \\
Science & $540 \mathrm{~s}$ & 1.079 & $2: 47$ & \\
Standard & $5 \mathrm{~s}$ & 1.258 & $2: 56$ & $b$ \\
Standard & $5 \mathrm{~s}$ & 1.246 & $2: 59$ & $b$ \\
Standard & $5 \mathrm{~s}$ & 1.064 & $4: 47$ & \\
Standard & $5 \mathrm{~s}$ & 1.064 & $4: 48$ & \\
Standard & $2 \mathrm{~s}$ & 1.063 & $4: 50$ & \\
\hline
\end{tabular}

Notes. HST: Hawaii standard time, ${ }^{(a)}$ expected fluxscale deviates from THELI's applied fluxscale by $\sim 28 \%,{ }^{(b)}$ large scatter in flux measurements of standard stars.

at $r_{\text {vir }}$ of $71 \pm 15 M_{\odot} / L_{\odot}$ (Khosroshahi et al. 2004). These data are consistent with the ROSAT study of Sanderson et al. (2003), but we note that their larger field-of-view constraints result in a slightly larger $r_{\text {vir }}$ and in $f_{\text {gas }} \approx 0.07$, which are more consistent with its $T_{\mathrm{X}} \sim 0.6 \mathrm{keV}$.

\subsection{Observations}

On 5 June 2008, $R$-band images of NGC 6482 were acquired using the Suprime-Cam wide field imaging instrument at the Subaru telescope (Table 1). The Suprime-Cam camera is a mosaic of ten $2 \mathrm{k} \times 4 \mathrm{k}$ CCDs with a pixel scale of $0.202 \operatorname{arcsec~pix}^{-1}$, and it covers an area of $34^{\prime} \times 27^{\prime}$ per field (Miyazaki et al. 2002). The field-of-view corresponds to a physical scale of $624 \times 458 \mathrm{kpc}$ at the distance of NGC 6482, reaching the virial radius at the field edges. Eighty-five per cent of the area that is enclosed by $r_{\text {vir }}$ is covered. After a chip replacement in July 2008, $B$-band images were obtained on 4 August 2008. Both observations were obtained in service mode under run ID S08B-150S (PI Hilker).

In either band, two short $(R: 60 \mathrm{~s}, B: 120 \mathrm{~s})$ and four long $(R: 540 \mathrm{~s}, B: 1020 \mathrm{~s})$ exposures were acquired, all centered on NGC 6482. The average seeing in the $R$-band was 0.6 arcsec FWHM and 1.0 arcsec in the $B$-band.

\subsection{Data reduction}

Suprime-Cam's reduction pipeline SDFRED was used to carry out overscan correction and flatfielding. As mentioned above, the $B$-band data were taken after a chip replacement. For this data set, each of the ten individual CCDs had four separate readouts, hence four different gains. Unfortunately, accurate individual gains were not provided with the data ${ }^{2}$. We adjusted the four gains within each CCD relative to each other such that sky brightness differences were less than $1 \%$ after the flatfielding step. This adjustment was determined with the long exposures for which gain variations dominate the relative count difference between the four stripes per chip. The relative gain corrections were then applied to all other $B$-band exposures (flats, standards, and short science exposures). The knowledge of the absolute gain value for the $B$-band data is not necessary because absolute

\footnotetext{
2 "For all data, S_GAIN[1-4] and GAIN values at FITS header have errors greater than $10 \%$. Those values are only for reference and should not be used for data analysis." (http://smoka.nao.ac.jp/help/ help_SUPnewCCD.jsp)
} 
Table 2. Photometric calibration parameters.

\begin{tabular}{lcc}
\hline \hline Filter & $R$ & $B$ \\
\hline$Z P[\mathrm{mag}]$ & $27.37 \pm 0.02$ & $27.07 \pm 0.06$ \\
$\kappa[\mathrm{mag}]$ & - & $-0.124 \pm 0.046$ \\
$X$ & $1.056 \pm 0.018$ & $2.006 \pm 0.318$ \\
$C T$ & $-0.001 \pm 0.019$ & $0.147 \pm 0.033$ \\
$A[\mathrm{mag}]$ & $0.22 \ldots 0.31$ & $0.35 \ldots 0.50$ \\
\hline
\end{tabular}

Notes. $Z P$ : zero point; $\kappa$ : atmospheric extinction coefficient; $X$ : mean airmass of exposures contributing to a coadded image; $C T$ : color term; A: Galactic extinction by Schlegel et al. (1998)

photometric calibration was achieved with the standard star exposures, themselves corrected with the same relative gain as the science data.

The THELI image reduction pipeline (Erben et al. 2005) then was used for the remaining steps of data preprocessing. The astrometric calibration from the THELI reductions was based on cross-correlation with the PPMXL catalog of point sources using the scamp software. It also corrected for geometric distortions in the outermost parts of the Suprime-Cam fields. After the THELI photometry step, which is based on SExtractor (Bertin $\&$ Arnouts 1996) and which adjusts the brightness levels of all chips and all exposures to each other, background subtraction was carried out using THELI.

After the THELI processing, instrumental magnitudes were computed from observations of standard stars taken in all four nights of the observing run, and from the photometry calibrated on the Cousins $B$ and $R$ magnitude system of Landolt (1992).

The average $1 \sigma$ noise per pixel for the 36-min coadded image in $R$-band corresponds to a surface brightness of $\mu_{R}=$ $27.2 \mathrm{mag} / \mathrm{arcsec}^{2}$, and $\mu_{B}=28.4 \mathrm{mag} / \operatorname{arcsec}^{2}$ for the 68 -minute composite image in the $B$-band. We thus have similar surface brightness sensitivities in $B$ and $R$, since typical early-type galaxy colors are around $B-R \lesssim 1.5 \mathrm{mag}$.

\subsection{Calibration}

The $R$-band and $B$-band data were observed on different nights. Each night, standard star fields were observed at two different points in time and for a range of different airmasses. At each airmass, two exposures per field were obtained. A significant extinction coefficient was found in the $B$-band, while for the $R$-band it was consistent with zero. Thus, the atmospheric extinction term in $R$-band is absorbed by the zero point (Table 2).

For the night of 5/6 June 2008 when $R$-band data were taken, there were unfortunately some transparency variations due to the presence of clouds (Table 2), which prompted us to take special care in the photometric calibration: the standard star exposures in the $R$-band around 03:00 Hawaii standard time (HST) showed huge sensitivity variations at an average zero point fainter than the mean of the other standard star exposures. We discarded these measurements and instead adopted the zero points measured two hours later around 05:00 HST, when conditions were stable. Fortunately, the $R$-band science images, taken two hours earlier, were obtained at almost exactly the same airmass, so that we do not introduce a luminosity offset when not considering the atmospheric extinction term. The observations of multiple standards throughout the night therefore allowed exclusion of those standard images with a notable drop in throughput. The used calibration parameters are displayed in Table 2. Systematic uncertainties due to photometric calibration arising from this table are given by $\sigma_{R}=0.04 \mathrm{mag}$ and $\sigma_{B}=0.12 \mathrm{mag}$ (uncertainties for Schlegel extinction are not provided).

We then proceeded to check for the presence of clouds in the $R$-band science data taken between 02:07 and 02:56 HST. In general, THELI compensates for varying atmospheric transmission during a sequence of images that are to be coadded. Relative flux offsets are determined and logged via the "fluxscale" parameter, from comparing the same sources in the individual images. All images are then normalized to the highest transmission within the stack of images. For a correct compensation of possible cloud effects, it is necessary to have at least one cloudless exposure in the stack of images that are coadded. THELI found that the first of the four long $R$-band exposures (540 s) taken at HST 02:11 was indeed affected by a significant flux drop ( 28\%) with respect to the other three long exposures. This was corrected by THELI in the final coadded image via the "fluxscale" parameter. We consider this correction as robust, since the other long exposures had relative fluxes consistent with each other at the $5 \%$ level.

The two short $R$-band exposures were taken immediately before the one long exposure that was apparently affected by reduced atmospheric transmission, between 02:07 and 02:11 HST. Among themselves, these short exposures (later used only for fitting the centers of the brightest galaxies) did not show any notable relative variation in the flux. However, given their fast cadence, this does not exclude that they were affected by clouds. To test for the presence of clouds, we ran SExtractor on both the short coadded image and the long coadded image, both normalized to $1 \mathrm{~s}$ integration time. The ratio of object fluxes between those two images shows that the short exposures had a $24 \%$ lower sky transmission than the long exposures. When we used the short time exposures for our analysis - only for the three brightest galaxies in our sample - we corrected the $R$-band flux by that offset factor of $1.31(=1 /(1-0.24))$. For consistency reasons, we applied the same procedure to the $B$-band data and found that the long time flux is $\sim 6 \%$ less than the short time flux. This difference is within the typical variation on a clear night, suggesting that no clouds were present during these observations. To be consistent with the treatment in the $R$-band, we corrected all $B$-band longtime fluxes upward by a factor of 1.06.

\section{Sample selection and photometric procedures}

\subsection{SExtractor and morphological classification}

SExtractor (Bertin \& Arnouts 1996) was used for detecting dwarf galaxy candidates in the field of view, followed by visual inspection. To optimize SExtractor's parameters, we simulated seeing convolved dwarf galaxies with exponential surface brightness (SB) profiles and circular morphology (ellipticity $=0$ ) and added them to the $R$-band Subaru field. We put only 50 artificial galaxies on randomly chosen fields of $1 \operatorname{arcmin}^{2}$ not to saturate the already crowded field with objects. That process was repeated 100 times. Later in this section, we also use these simulations for completeness determination.

Dwarf galaxies are found to be relatively homogeneous in terms of their photometric scaling relations (Misgeld \& Hilker 2011) among different environments. As input for our simulations, we used the $\mu$-mag relation found by Misgeld et al. (2009) for dwarfs in the Centaurus cluster ${ }^{3}$ with a simulated scatter in $\mu$ of $\pm 0.96 \mathrm{mag}$ around the fiducial relation. This

\footnotetext{
$\mu_{V, 0}=0.57 \cdot M_{V}+30.90$ and converted to $R$-band by adopting $V-R=$ $0.6 \mathrm{mag}$.
} 


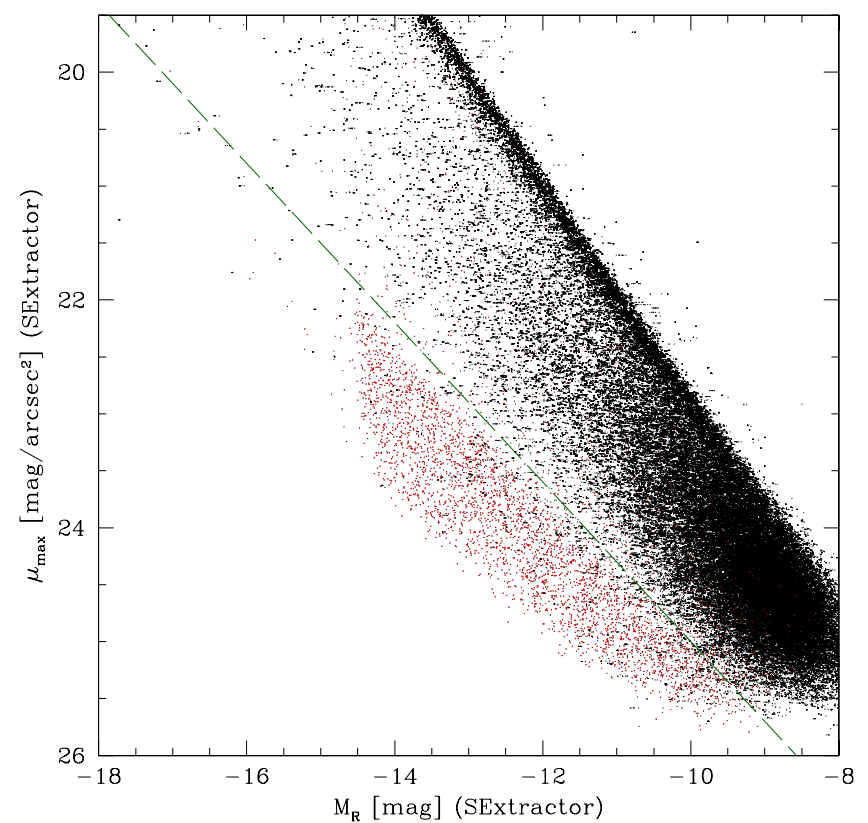

Fig. 1. SExtractor MAG_BEST and peak surface brightness for all nonsaturated objects detected in the long exposure of the Subaru NGC 6482 field of view, including simulated dwarf galaxies with apparent sizes corresponding to the distance of NGC 6482. The SExtractor object detection parameters are given in Table 3. Black dots: all detected objects. Red dots: discovered simulated dwarf galaxies. Green dashed line: separation line adopted between galaxies and unresolved objects at the faint end $\left(\mu_{\text {peak }}=30+0.7 M_{R}\right)$.

scatter corresponds to the $2 \sigma$ width of the relation found by Misgeld et al. (2009). The goal of this exercise is to determine the location of typical dwarf galaxies in SExtractor's MU_MAX-MAG_BEST space, in order to establish a distinction from the crowd of small faint objects whose apparent sizes are close to the resolution limit of our data (Fig. 1). The parameter space occupied by the simulated dwarf galaxies is clearly offset from the bulk of faint sources, which allows defining a fiducial separation line as indicated in Fig. 1. All objects below the green line in that plot are considered as possible members of the NGC 6482 group. A small minority of simulated dwarf galaxies have recovered SExtractor parameters above that line, because they are superposed on another object - mostly a brighter foreground star.

Overall, the analysis of SExtractor's findings showed that even with extreme settings, only up to $80-85 \%$ of the simulated galaxies for the brightest galaxy bin are discovered (see Fig. 2). This is due to a crowding completeness limit imposed by the high foreground star density towards NGC 6482 owing to its low galactic ( $l=48, b=23$ ) latitude (see, e.g., Fig. 3 ). In the halo of bright stars, an automated detection algorithm like SExtractor tends to "overlook" individual sources. In addition to the crowding incompleteness, the usual surface-brightness incompleteness begins at $M_{R} \sim-13$ mag. We used an analytical expression to describe the surface brightness incompleteness, normalized to the crowding incompleteness level. This function is shown in Fig. 2. It reaches a 50\% completeness at $M_{R} \approx-10.5 \mathrm{mag}$ and was used to correct the luminosity function (LF) for incompleteness.

The SExtractor detection parameters were varied (see Fig. 2 and Table 3) in order to find the optimal set in terms of recovered simulated galaxies with respect to all detections classified as galaxy - given in Fig. 1. We stress that for the

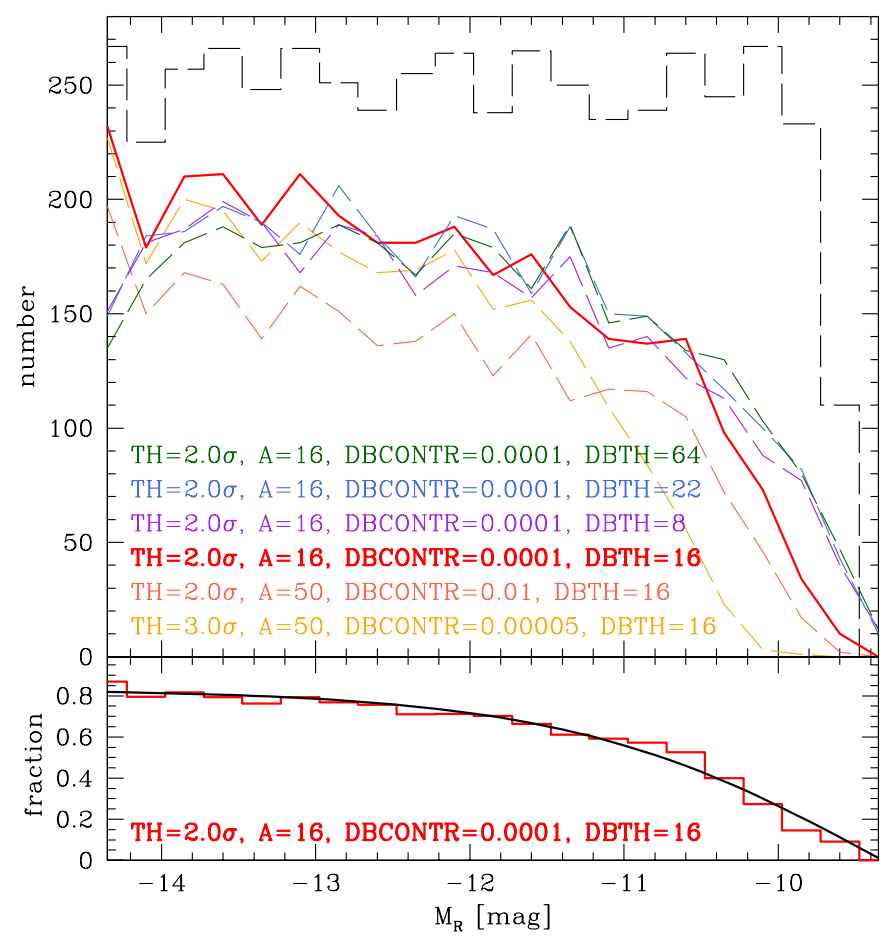

Fig. 2. Determination of optimal SExtractor parameters. Upper panel: selection of tested SExtractor parameter sets. Colored curves show SExtractor detection number counts for different setups (TH: detection threshold; A: minimum area of pixels above threshold; DBTH: number of deblending subthreshold; DBCONTR: deblending contrast). The black, dashed histogram indicates the number of simulated galaxies in each magnitude bin. The red solid line represents our final choice, since it provides an optimal balance between the fraction of recovered objects and the detection of spurious ones. Lower panel: the red, solid histogram shows the fraction of recovered simulated galaxies using the optimal parameter set. The black solid line is a fit to the red histogram (i.e., our completeness function). Owing to crowding incompleteness (high foreground star density), the recovery completeness saturates at $80 \%$ for the brightest sources.

Table 3. Optimized SExtractor parameters for dwarf galaxy detection.

\begin{tabular}{lc}
\hline \hline Parameter & Value \\
\hline DETECT_MINAREA & 16 \\
DETECT_THRESH & $2.0 \sigma$ \\
DEBLEND_NTHRESH & 16 \\
DEBLEND_MINCONT & 0.0001 \\
BACK_SIZE & 12 \\
BACK_FILTERSIZE & 11 \\
BACKPHOTO_TYPE & GLOBAL \\
\hline
\end{tabular}

Notes. This parameter set is sensitive to faint extended objects but many more unresolved objects are found. Photometric output parameters are reliable in general, but individual outliers of up to $0.5 \mathrm{mag}$ between input and recovered magnitude can occur due to the rather extreme setting of background determination.

actual photometry of dwarf galaxy candidates, individual aperture photometry was performed. The automatic magnitudes measurements by SExtractor are only used for the preselection of probable dwarf galaxy candidates. The optimal SExtractor parameter set for dwarf galaxy detection is displayed in Table 3, and yielded a total of 621 galaxies below the green line, out of a total of 61120 detections in the field of view. Those 621 objects were a preselection and were subsequently inspected visually by 


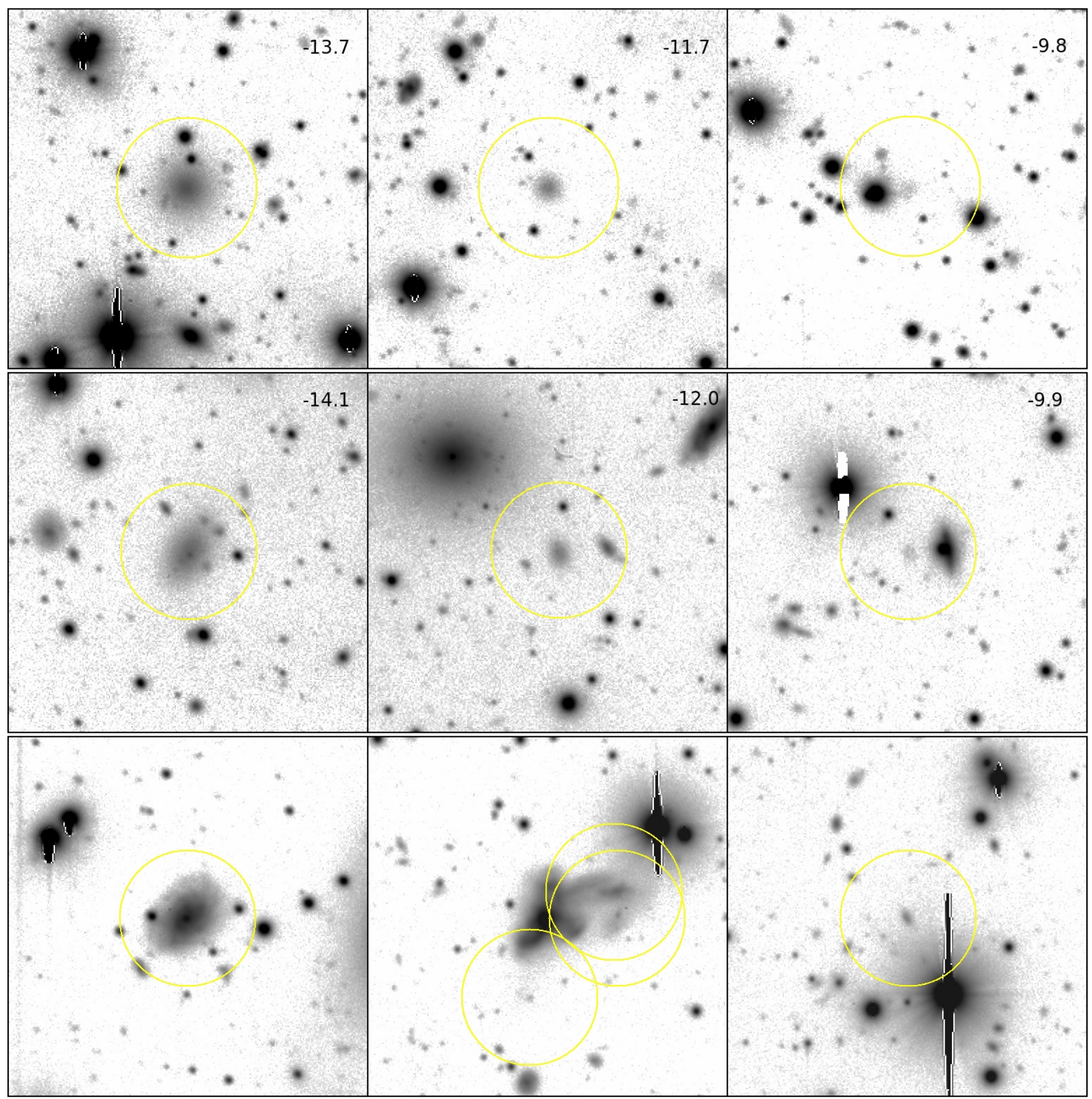

Fig. 3. Illustration of example SExtractor detections. In each case the yellow circle has a diameter of 20 arcsec and is centered on the object in question. Numbers in the upper right corner denote absolute $R$-band magnitudes. Upper panel: simulated galaxies placed into deep science images. Middle panel: objects accepted as possible members after visual inspection. Lower panel: rejected objects because of compact appearance or visible structure typical of background grand-designed spirals or mergers.

two of the authors (SL and SM) in an independent manner in order to reject artifacts (see Fig. 4). The majority of sources were indeed readily identified artifacts in the halos of bright stars, see e.g., the lefthand panel of Fig. 4. Another goal of the visual inspection was to reject obvious background galaxies, such as low surface-brightness spiral galaxies, interacting low surfacebrightness galaxies (merging events), or small, very faint galaxies that appear too compact (compare right center panel and right bottom panel of Fig. 3). In particular, the last distinction has an impact on the number counts at the faint end of our sample. It is well known that faint dwarf galaxies (at $M_{R} \gtrsim-12 \mathrm{mag}$ ) are diffuse, while their effective radius does not change significantly with luminosity (see Misgeld \& Hilker 2011). As a result, we implicitly assume for our visual inspection that the faint dwarf galaxies should exhibit such a diffuse appearance.

Of the 621 SExtractor detections, 83 remained as visually confirmed candidate dwarfs. Twelve other sources were rejected because either a spike of a nearby foreground star covered the object's center, a structure was visible after the subtraction of the modeled galaxy (see Sect. 3.2.1), or there was no counterpart found in $B$-band, or only a very weak one ( $\sim \sigma$ above sky). Furthermore, two obvious dwarf galaxies (with $M_{R} \sim-11.5 \mathrm{mag}$ and $-10.7 \mathrm{mag}$ ) not detected by SExtractor and all bright galaxies (six galaxies brighter than $M_{R}=-18 \mathrm{mag}$ and a diffuse 

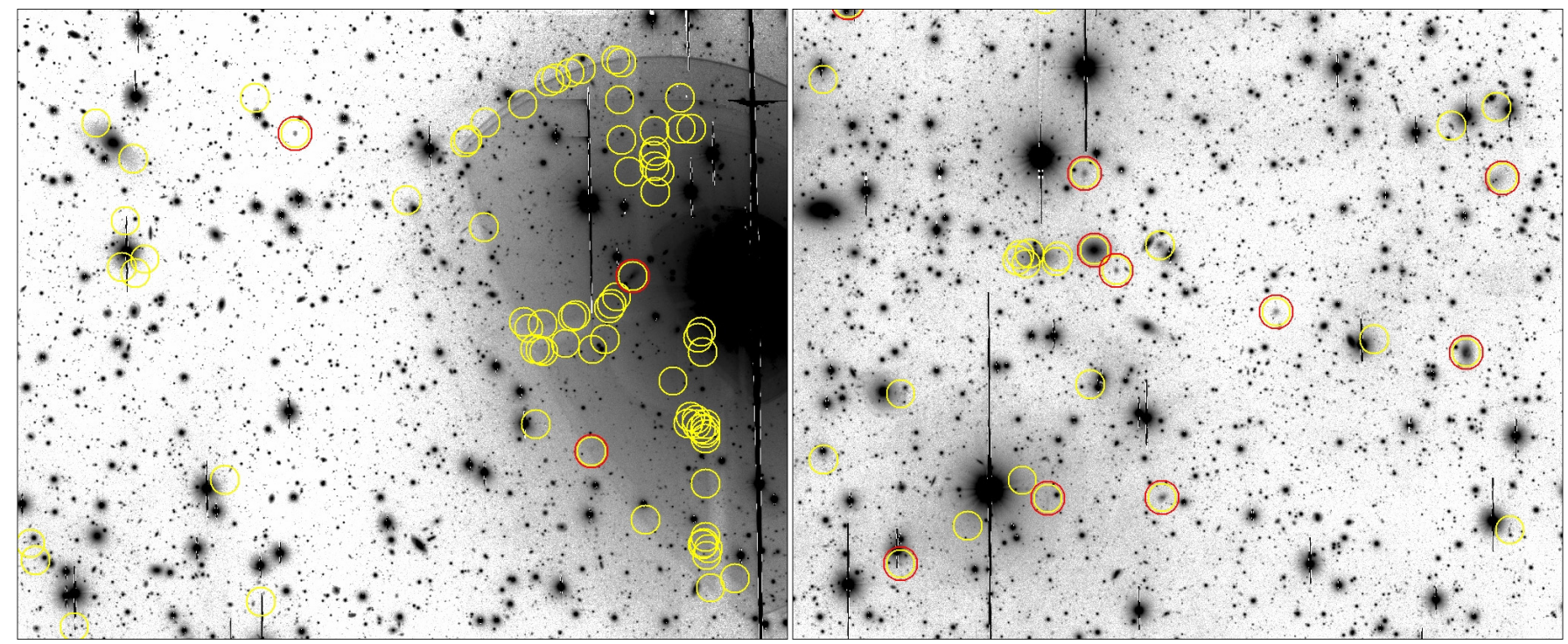

Fig. 4. Illustration of preselected SExtractor detections considered as possible members (red circles) and artifacts/background sources (yellow circles). Left: many objects are rejected since they are detected in the refraction halo of a foreground star and in the shadows along instrument suspension. Right: a field not affected by artifacts where detections close to spikes of foreground stars were rejected. Only a few objects remain and are considered as possible group members (red circles).

dE with $M_{R} \sim-15.6$ mag) were included in the sample since SExtractor was tuned to find faint galaxies with $M_{R}>-14$ mag. Overall, 80 galaxies in the absolute magnitude range $-8.8 \mathrm{mag}$ to -22.7 mag at NGC 6482's distance were selected for detailed photometric analysis.

The morphological classification we adopted during visual inspection follows the extended Hubble scheme by Sandage \& Binggeli (1984). In the dwarf regime, we simplify it by labeling early-type dwarf galaxies generally as " $\mathrm{dE}$ " and irregular dwarf galaxies as "dIrr". Examples of our classification are shown in Fig. 5. The morphological classification type of each group galaxy is provided in Table A.1.

\subsection{Color-magnitude diagram}

The morphologically preselected sample was cleaned of further probable nonmembers via color selection criteria. In the following Sect. 3.2.1, we describe the photometric procedures applied for the color measurement and in 3.2.2 discuss the selection of the fiducial sample based on the distribution in color-magnitude space.

\subsubsection{Photometric procedure for magnitude and color measurement}

To correct for the light blurring due to the PSF, we degraded the $R$-band images to the worse seeing of the $B$-band images (1.0 arcsec) using IRAF task psfmatch. By doing this, we expected to measure the flux within the same physical isophotes. Since the observed field is very crowded, the light of a very bright foreground star's reflection halo partially contaminates the light of a selected dwarf galaxy in many cases (see, e.g., left image of Fig. 4). In those cases the star was modeled in both passbands (using ellipse) and subtracted from the image in order to obtain more reliable values for the galaxy's luminosity. All galaxies were photometrically analyzed using the ellipse task (Jedrzejewski 1987) that is included in the STSDAS package of IRAF. All ellipse fits were performed with fixed parameters for center coordinates and position angle but variable ellipticity. In some cases, like the central galaxy NGC 6482 itself, optimal results were obtained when the position angle was allowed to vary. Because the seeing of the $R$-band images was better, we did a first ellipse run for the undegraded $R$-band images. Those were used for the surface brightness analysis in Sect. 3.3. Another ellipse run was performed to fit the degraded $R$-band images. We then applied the obtained isophote table to the $B$-band image of the same object to measure the flux within the same physical isophotes. Obvious faint foreground stars and background galaxies were masked, and ellipse fits were performed far beyond the galaxy's edge to see whether the signal reaches the amplitude of the background noise. Using that level, an individual background adjustment was done for every galaxy. The result of this background estimation is shown in the three panels of Fig. 6. The intensity levels out at zero and associated error bars become as high as the signal; i.e., the signal is dominated by background noise (note the logarithmic scale of the intensity - right axis).

The ellipse output tables were used to determine all astronomical quantities that are presented in this study. The truncation radius of a galaxy was defined to be the last isophote at which the intensity is still higher than its error. The radial profiles of the cases shown in Fig. 6 are displayed out to that truncation radius. The flux enclosed by that ellipse is used as (measured) total flux $f$. Using that flux the apparent magnitude of an object is calculated. Finally, the apparent magnitude of an object was corrected for galactic extinction by applying the foreground extinction map of Schlegel et al. (1998). In Table 2 quantities determined for the photometric calibration are listed. The photometrical uncertainties of the dwarf spheroidals (dSph) are dominated by sky noise.

The half-light radius $r_{50}$ is determined as the radius enclosing $50 \%$ of the measured total flux $f$. We determine the mean $B-R$ color of each object using the flux within $r_{50}$ as determined in $R$-band. Within $r_{50}$ the signal-to-noise ratio is higher than for the total flux. In light of the crowded field and the transparency variations (see Sect. 2.4) in some cases a variable sky 


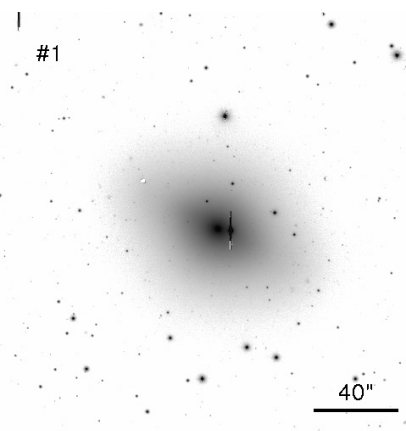

(a) $\mathrm{E}$

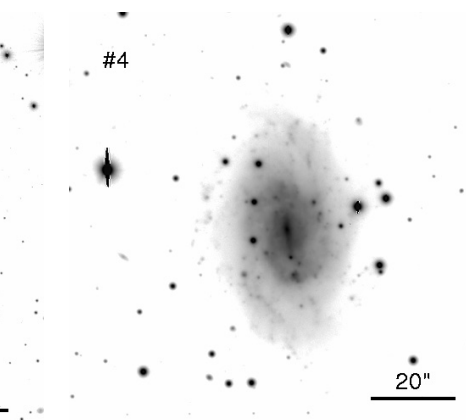

(b) $\mathrm{SBc}$

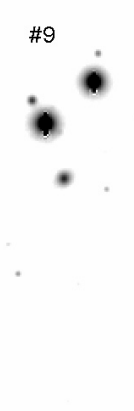

(c) dIrr

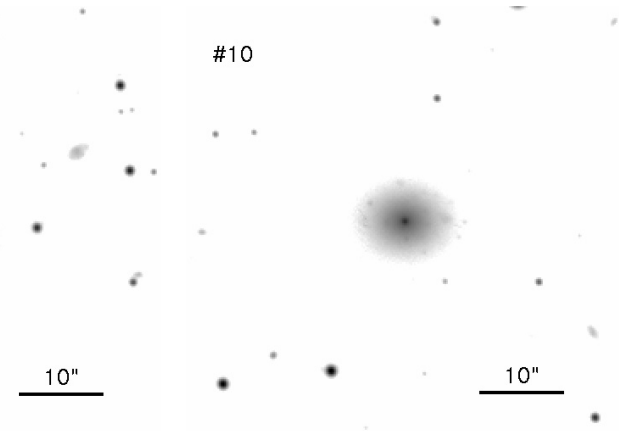

(d) $\mathrm{dE}, \mathrm{N}$

Fig. 5. Examples of morphological classification (provided in subcaptions).
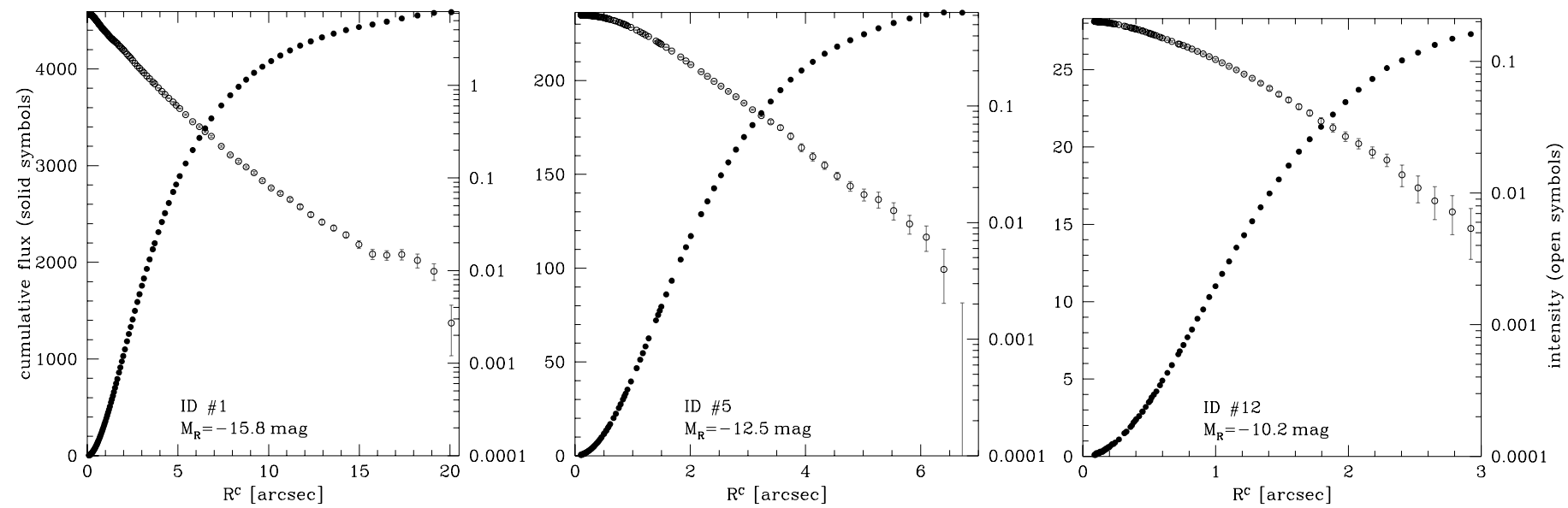

Fig. 6. Intensity profiles (open symbols, right $y$-axis) and cumulative flux (solid symbols, left $y$-axis) of three arbitrarily chosen galaxies in the sample. Shown are all data points used for determining the total flux.

background can occur, making $r_{50}$ more reliable for our color estimation, especially for very low surface-brightness targets for which total fluxes are difficult to determine.

\subsubsection{Fiducial sample definition via color-magnitude selection}

The colors of all objects with $B-R<1.75 \mathrm{mag}$ and brighter than $M_{R}=-10.5 \mathrm{mag}$ are shown in the color-magnitude diagram (CMD) in Fig. 7. We consider redder galaxies to be background contamination ${ }^{4}$. Galaxies fainter than this limiting magnitude have very large $B-R$ color errors and are in the luminosity regime where the detection completeness is below $50 \%$. The colors represent the integrated value within the half-light radius $r_{50}$. Only the color of the BGG, whose inner arcsec is saturated in $R$-band, is represented by its value at $r_{50}$. The $B-R$ value of the disrupted galaxy (at $M_{R} \approx-17.7 \mathrm{mag}$ ) comes from SExtractor analysis. It is clearly visible in the plot that three disky galaxies fall almost exactly on the red sequence (RS hereafter). These are the S0 host of the disrupted galaxy, an S(lens)0 and a dusty edgeon spiral - the bright blue square in Fig. 7. See ID 3, 6, and 2 in Table A.1. The sample also contains two blue, almost face-on

\footnotetext{
4 A 12-Gyr-old stellar population with super-solar metallicity $([\mathrm{Fe} / \mathrm{H}]=+0.2 \mathrm{dex})-$ typical of luminous early-type galaxies - has a $B-R$ color of $\sim 1.75 \mathrm{mag}$ (Worthey 1994).
}

spirals and three dIrrs denoted by filled blue squares. The four brightest galaxies are spectroscopically confirmed group members (and indirectly the disrupted galaxy, see Sect. 4.5). Nicely visible in the CMD is the two-magnitude gap in $R$-band between the BGG and the second-ranked galaxy - one of the fossil criteria. Also noticeable is that the second brightest early-type galaxy has $M_{R}=-17.7 \mathrm{mag}-$ five magnitudes fainter than the BGG, already entering the dwarf galaxy regime.

To improve on the purely morphological constraints of group membership described in the previous section, we color-restrict our sample to galaxies that lie within $3 \sigma$ of the red sequence (RS), whose location we determine with a least square fit to the data points shown in Fig. 7. Not to be affected by the larger scatter of faint galaxies we fit the RS only to galaxies brighter than $M_{R}=-14.0 \mathrm{mag}$, and exclude the obvious blue sequence objects, as well as three photometrically uncertain galaxies.

We obtain the following least-square fit for the colormagnitude relation (CMR) of the RS in the NGC 6482 group

$B-R=(-0.029 \pm 0.008) M_{R}+(1.05 \pm 0.13)$

with an rms of $0.06 \mathrm{mag}$. The index $r_{50}$ given to the color legend $(B-R)$ on the $y$-axis indicates that the values are the average within the half-light radius. The CMR fits the relation defined by the brightest early-type galaxies in our sample well, down 


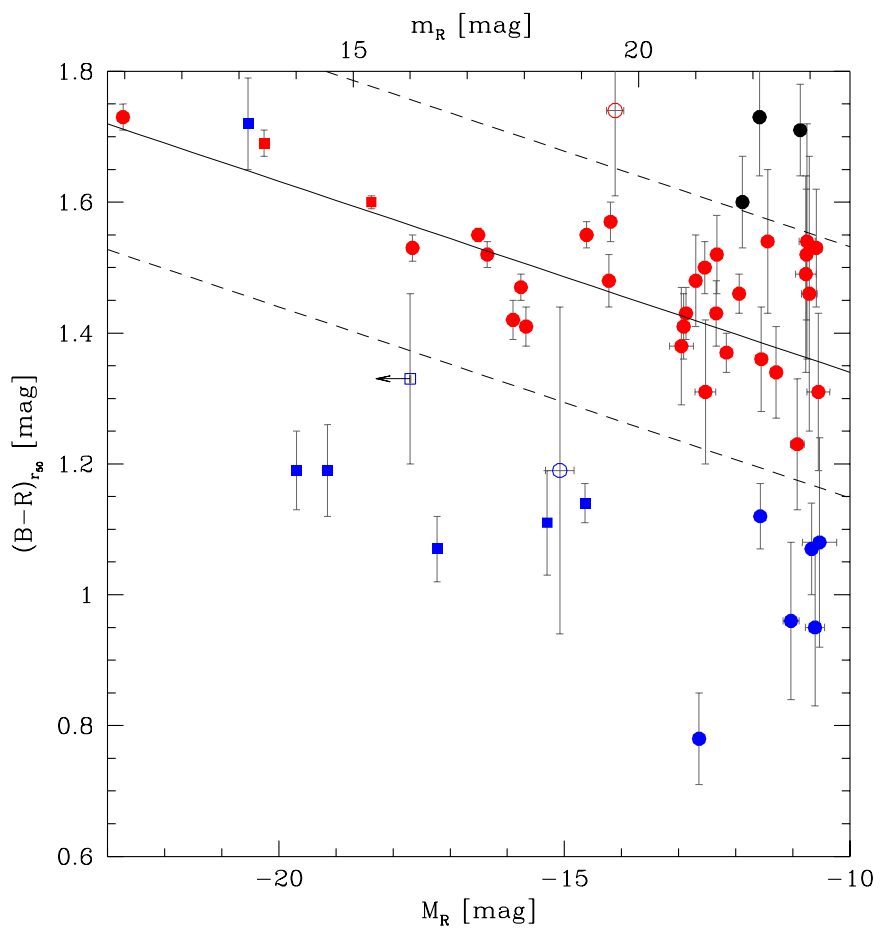

Fig. 7. Color-magnitude diagram of all objects brighter than $M_{R}=$ $-10.5 \mathrm{mag}$ (our $50 \%$ completeness limit) and bluer than $B-R=$ 1.75 mag. $B-R$ colors represent the integrated value within the halflight radius determined in $R$-band, except for the BGG - where the color is determined at $r_{50}$-, and the disrupted galaxy (see Sect. 4.5). Red data points denote red sequence galaxies that we consider to be group members (circles: elliptical galaxies, squares: S0 galaxies), blue data points for blue cloud galaxies (circles: blue dSphs, squares: spirals and irregulars), respectively. Black data points are galaxies considered to be background galaxies. The open blue square denotes the disrupted galaxy of Sect. 4.5. Open circles (one blue, one red) represent galaxies with uncertain photometry because the galaxy is superposed on a brighter object (BGG or refraction halo of a star) that could not be fully modeled. Because of their morphology, those galaxies are included in the sample. The solid black line is a best fit for our red sequence CMR for galaxies brighter than $M_{R}=-14$ mag (see text). The dashed lines illustrate the $3 \sigma$ level of confidence, which is used to reject background galaxies from the sample on the red side.

to $M_{R} \sim-14 \mathrm{mag}$, and is also consistent with the color distribution of the fainter galaxies - even though these exhibit a larger scatter. In the following, every object within the $3 \sigma$ RMS of the fit and brighter than $M_{R}=-10.5 \mathrm{mag}$ is considered to be an RS member of the group. We furthermore include two galaxies in this fiducial sample with uncertain photometry that formally places them redward of the above $3 \sigma$ range, but they morphologically resemble diffuse dwarf galaxies at the group's distance $^{5}$ (see also Fig. 8). Galaxies belonging to the blue cloud are labeled with blue datapoints. After selection around the red sequence, twelve out of 80 galaxies were considered to be background galaxies on the basis of their extreme red colors. Three of those are visible as black datapoints in the CMD. When only considering galaxies brighter than the $50 \%$ completeness level at $M_{R}=-10.5 \mathrm{mag}, 22$ further galaxies are disregarded. Finally, including the two probable members with uncertain photometry mentioned above, this yields a fiducial sample of 48 probable group member galaxies (see Table A.1).

\footnotetext{
5 These galaxies are listed in Table A.1 with IDs $20\left(M_{R}=14.2 \mathrm{mag}\right.$, $\left.B-R=2.48_{-1.13}^{+0.12} \mathrm{mag}\right)$ and $22\left(M_{R}=14.1 \mathrm{mag}, B-R=1.74 \pm\right.$ $0.13 \mathrm{mag})$.
}

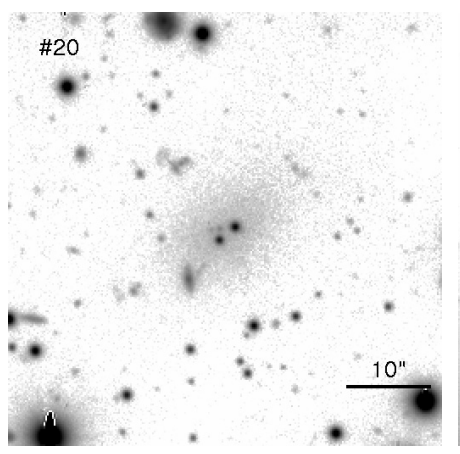

(a) $\mathrm{dE}(, \mathrm{N}$ ?)

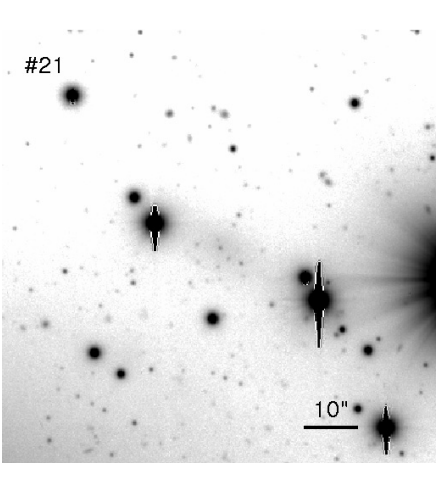

(b) dE,N
Fig. 8. Galaxies with uncertain photometry due to BGG's halo and several stars in close proximity. We believe both galaxies are part of the group. In the right panel, we could not account for additional brightness gradients from the BGG and a very bright foreground star.

\subsection{Surface brightness profile measurements}

Surface brightness (SB) profiles of all investigated galaxies were analyzed using the analytic expression suggested by Sérsic (1968). In our case we fitted single Sérsic profiles to the $R$-band SB obtained from the undegraded images (see Sect.3.2.1), that is,

$\mu\left(R^{\mathrm{c}}\right)=\mu_{\mathrm{e}}+1.0857 b_{n}\left[\left(\frac{R^{\mathrm{c}}}{r_{\mathrm{e}}}\right)^{1 / n}-1\right]$,

where $\mu_{\mathrm{e}}$ is the $\mathrm{SB}$ of the isophote at the effective radius $r_{\mathrm{e}}$. The constant $b_{n}$ is defined in terms of the parameter $n$ that describes the shape of the light profile. As shown by Caon et al. (1993), a convenient approximation relating $b_{n}$ to the shape parameter $n$ is $b_{n}=1.9992 n-0.3271$ for $1 \lesssim n \lesssim 10$, which we applied in our calculations. The $\mathrm{c}$ in the variable $R^{\mathrm{c}}$ denotes that we performed the Sérsic fits with respect to the circularized radius $R^{\mathrm{c}}=a \sqrt{1-\epsilon}$, where $a$ is the major axis of the isophote with its ellipticity $\epsilon$. We note that a multiple Sérsic fit would be more appropriate in the case of the $\mathrm{S}$ (lens) 0 galaxy in our sample (ID 6 in Table A.1) (see Kormendy \& Bender 2012 and Janz et al. 2012). Thus, the errors of a single Sérsic fit are rather large for this galaxy. For consistency with the rest of the sample, we stick to a single fit as reference. We note at this point the importance of a reliable background estimation for the SB fits to dwarf galaxies. Caon et al. (2005) show that incorrect sky background estimates lead to significant differences in the Sérsic fitting parameters. We are confident that the individual curve-of-growth method described above gives a robust background estimate for each galaxy.

The Sérsic fits were performed with different fitting ranges. The standard fit excluded the inner two arcseconds (i.e., one arcsecond of $R^{\mathrm{c}}$ ), which is about three times the seeing, and was performed until the intensity reaches $\mu_{R}=26.5 \mathrm{mag} / \operatorname{arcsec}^{2}$. For some very faint $\mathrm{SB}$ profiles, the fit did not converge so that either only the inner arcsecond was excluded or the limiting SB was set to $\mu_{R}=25.0 \mathrm{mag} / \operatorname{arcsec}^{2}$. Nonetheless, we tried to perform the fit with all of the three settings to get robust error estimates that are provided in Table A.1. For nucleated galaxies, only the main body of the galaxy was fitted, excluding the central luminosity spike. Total luminosities were not computed from those fits, but from information of the total galaxy flux given by the ellipse outputs. 

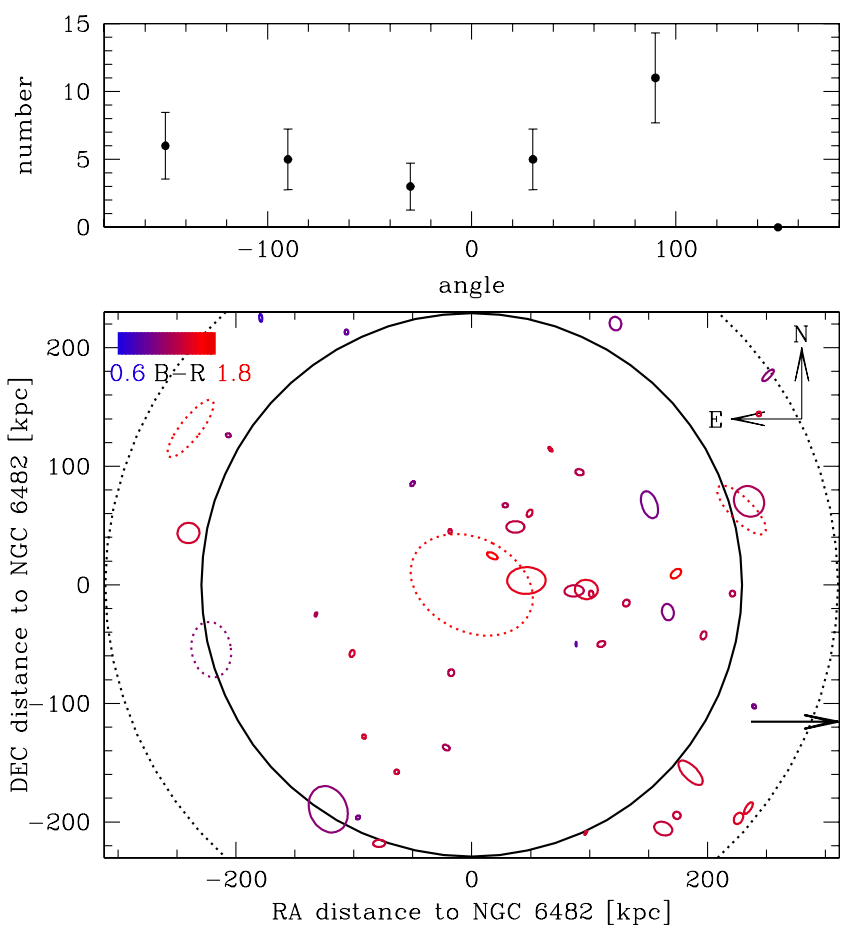

Fig. 9. Lower panel: spatial distribution of all galaxies considered group members (blue and red sequence, see Sect. 3.2.2). Spectroscopically confirmed group members are denoted by dotted shapes. All objects are color-coded with respect to their $B-R$ color, and the size of each galaxy is scaled to its luminosity by roughly $L^{0.03}$. Ellipticity and position angle are represented by its value at the half-light radius. The solid circle represents the area that is completely covered by our field of view $(r=229 \mathrm{kpc})$. The size of the dotted circle corresponds to the virial radius $\left(r_{\mathrm{vir}}=310 \mathrm{kpc}\right)$. The arrow in the southwest corner indicates that another spectroscopically confirmed cluster member lies outside the field of view. Upper panel: angular distribution of all galaxies shown in the lower panel within the solid circle. The 0 deg position is north in the lower panel and the angle grows clockwise. The errors in this plot are Poissonian.

The results of these measurement are used to analyze the photometric scaling relations of dwarf galaxies in the fossil group NGC 6482, and to identify galaxies in our sample that exhibit centrally concentrated light profiles - typical of intrisically luminous galaxies - resulting in high Sérsic $n$ (more than 2). In particular for galaxies with faint total magnitudes, this would indicate that they are background galaxies following Lieder et al. (2012).

\section{Results}

That galaxies are brighter in the $R$-band than in the $B$-band $(\sim 1.5 \mathrm{mag})$ roughly compensates for the missing depth in the $R$-band ( $\sim 1.2 \mathrm{mag})$. Since the seeing is better in the $R$-band, we present all results related to the according $R$-passband quantities.

\subsection{Spatial distribution}

In Fig. 9 we present a $B-R$ color-coded, luminosity-scaled spatial distribution of all galaxies within the field of view that are considered group members. There is another spectroscopically confirmed group member outside the field of view with an apparent $B$-band magnitude of $m_{B}=15.5$ mag (Zwicky et al. 1963) - comparable to the other spirals in this study.

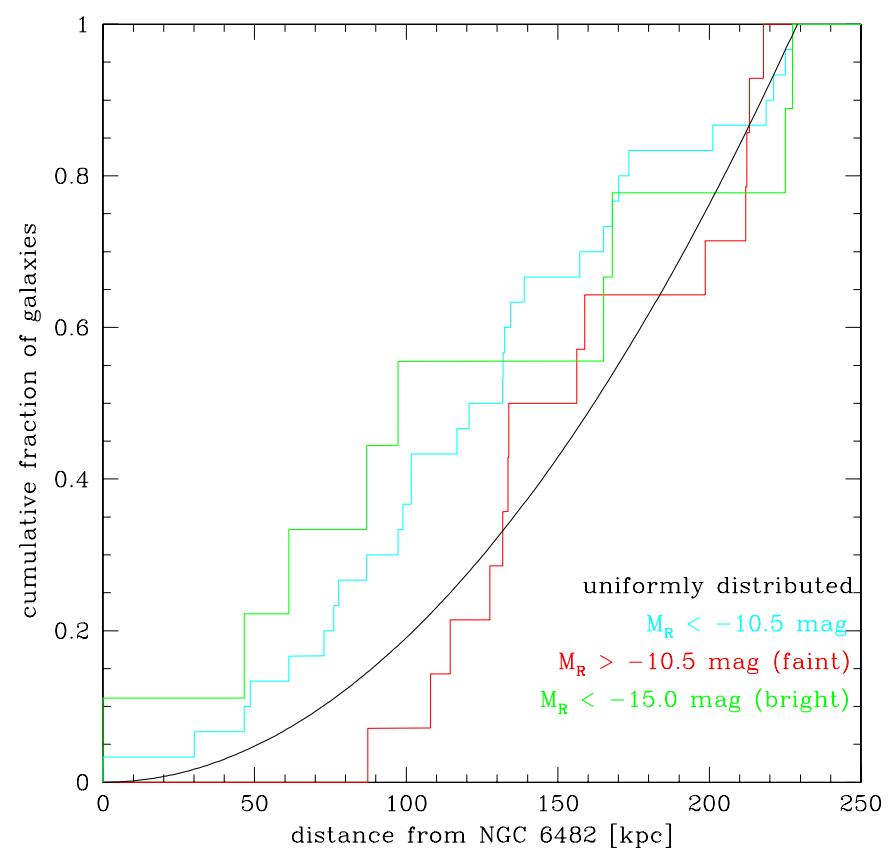

Fig. 10. Radial distribution of investigated galaxies represented by their cumulative fraction with respect to their distance to the BGG. Only galaxies within the solid circle in Fig. 9 (largest completely covered annulus) are taken into account as long as they obey the membership constraints of Sect. 3.2.2. Green: bright galaxies $\left(M_{R}<-15\right.$ mag). Red: faint galaxies $\left(M_{R}>-10.5 \mathrm{mag}\right)$. Light blue: all galaxies brighter than $M_{R}=-10.5 \mathrm{mag}$. The black solid line represents a uniform distribution as would be the case for background galaxies.

The investigation of the angular distribution of the group galaxies (only within the fully covered circle of $229 \mathrm{kpc}$ ) shows a preferred location of galaxies towards the west $\left(90^{\circ}\right.$ position; see upper panel of Fig.9). There are five galaxies in the far southwest end whose projections look very clustered. Those five galaxies may constitute an intruding subgroup.

Another constraint on the group membership of the mentioned galaxies arises from Fig. 10. There we plot the cumulative radial distribution of galaxies of certain magnitude intervals with respect to their distance from the BGG, to test whether they are clustered towards the BGG. From this plot it is evident that the bright galaxies $\left(M_{R}<-15 \mathrm{mag}\right)$ are concentrated around the BGG as compared to an uniform distribution of galaxies. This also holds for all galaxies we suppose to be group members.

\subsection{Photometric scaling relations}

In Fig. 11 we present the most relevant photometric scaling relations of the galaxies presented in the CMD and the additional probable member with uncertain photometry at $B-R=2.48$, ID 20 in Table A.1, including effective SB $\mu_{\mathrm{e}}$ at the effective radius $r_{\mathrm{e}}$, effective radius, Sérsic index $n$, and total luminosity. The top panel in particular shows the correlation between effective SB and effective radius, also known as the Kormendy relation (Kormendy 1977). We stress here that all quantities except the total magnitude are values that arise from single Sérsic fits to the light profiles of the undegraded images (see Sect. 3.2.1) down to $\mu_{R}=26.5 \mathrm{mag} / \operatorname{arcsec}^{2}$. We disregard central bright components like nuclei; i.e., only the main body of the galaxy is considered. Galaxies whose properties constitute strong outliers in these plots are typically candidates for background galaxies. There is one source that is an outlier in three of the four plots: this is the 


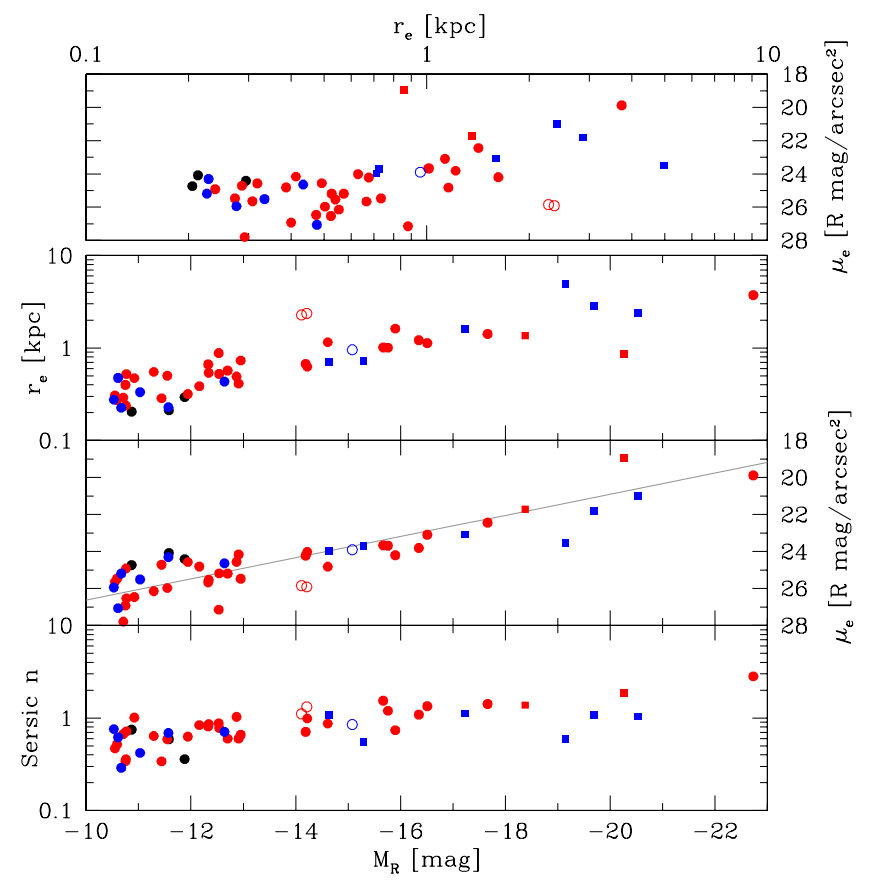

Fig. 11. Photometric scaling relations of all investigated galaxies. Except for the total magnitude (curve-of-growth), all quantities arise from single Sérsic fits. In the case of spiral galaxies, the fit was performed to the disk; for dE,N only the main body was fitted. Symbols as in Fig. 7. While $r_{\mathrm{e}}$ is the effective radius, $\mu_{\mathrm{e}}$ represents the effective SB (at $r_{\mathrm{e}}$ ). The gray line in the $\mu_{\mathrm{e}}-M_{R}$-plot gives the relation of Misgeld et al. (2009). The disrupted galaxy is not shown in this plot (no light profile available).

S0 galaxy (MRK 0895), the host of the disrupted galaxy (see Sect. 4.5), a spectroscopically confirmed cluster member. It has a comparatively high surface brightness and small size compared to the main body of group member galaxies that might be related to the edge-on view, which is simply an effect of its high inclination (see Fig. 14). We note that this galaxy is well fit by a single Sérsic profile (as seen in the right panel of Fig. 14). That the grand design spiral galaxies tend to deviate from the photometric scaling relations given by the early types is also visible in these plots. The spirals tend to have larger half-light radii and fainter effective SBs as expected from the early-type relations, resulting in lower concentration parameter $n$. This is expected since our Sérsic fits consider the disks alone. Two other outliers represent the probable members with uncertain photometry. These are the galaxies displayed in Fig. 8. For the galaxy with two foreground stars in the center, the low SB can be due to difficult masking that took most of its light.

Clear trends are visible in all relations in the sense that brighter galaxies are larger $\left(r_{\mathrm{e}}\right)$ and have brighter SB at the half-light radius and more centrally concentrated light profiles (Sérsic $n$ ). There is no faint galaxy with high Sérsic $n$ that would qualify it as background galaxy. This is a consequence of our applied $\mu$ - mag selection criterion (see Sect.3.1), which initially rejects faint galaxies with high central light concentration. Another point is worth mentioning. We do not see any galaxy within the interval $-14<M_{R}<-13$ mag. A similar dip in the galaxy luminosity function around this magnitude was reported by Hilker et al. (2003) for the Fornax cluster. We, however, refrain from addressing the statistical significance of the gap and its origin owing to the low number statistics at these low luminosities.

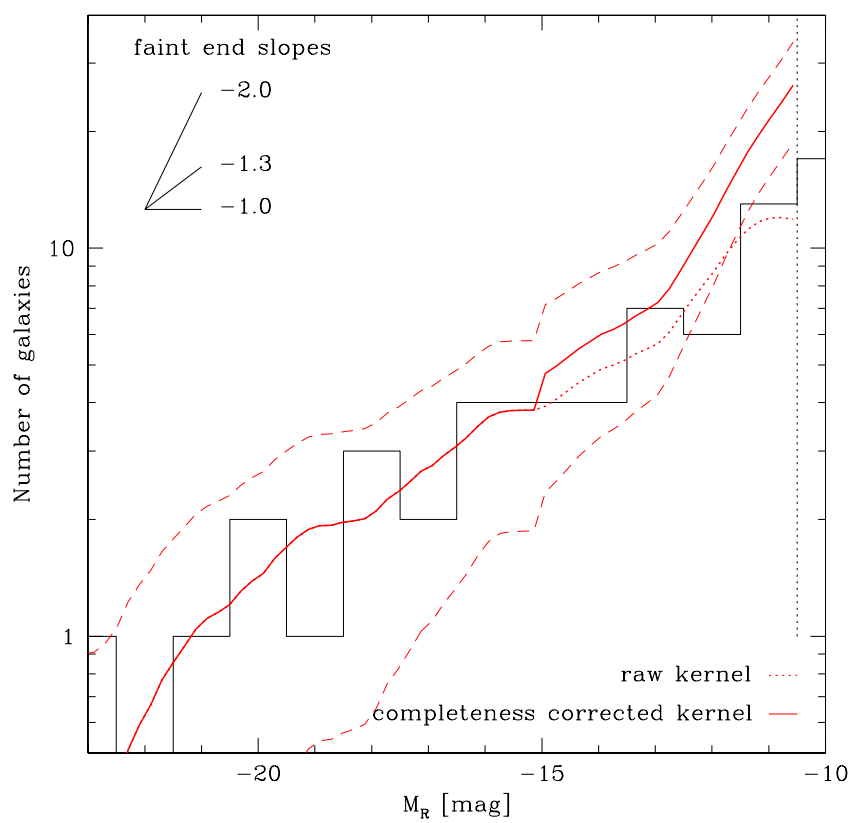

Fig. 12. $R$-band luminosity function of members considered galaxies (see Fig. 7). The black histogram represents the observed data (bin width: $1 \mathrm{mag}$ ). The red dotted line is a binning-independent representation of the counts (Epanechnikov kernel of 1 mag bin width), while the red solid line is its completeness-corrected (see Sect. 3.1) counterpart with the $1 \sigma$ uncertainty limits (dashed). The vertical dotted line is our 50\% completeness limit at $M_{R}=-10.5 \mathrm{mag}$. For comparison, some faint end slopes are illustrated in the top left corner of the plot. The best fit slope to our data is $\alpha \sim-1.3$, see text.

\subsection{Luminosity function}

In Fig. 12 we show our completeness uncorrected galaxy luminosity function (1 mag bin width, steps of $1 \mathrm{mag}$ ). The sample used for the LF are the 48 galaxies considered as likely members, see Sect. 5.2. For a better visualization of the LF we use a binning-independent sampling of the completeness-corrected LF, performed by an Epanechnikov kernel (Epanechnikov 1969) with a bin width of $1.0 \mathrm{mag}$ - displayed with red colors in Fig. 12.

Because of the missing $L^{*}$ galaxies in a FG, the bright end of the LF looks different from normal cluster LFs. Thus, a Schechter fit to the LF will only be poorly constrained at the bright end. Nevertheless, it is meaningful for the faint end. We performed a fit to the galaxy number count distribution (assuming Poissonian errors), including completeness correction for galaxies fainter than $M_{R}=-15$. The fitting interval was chosen to end with $M_{R}=-10.5 \mathrm{mag}$ (our $50 \%$ photometrical completeness limit). We fit the number count distribution of all galaxies in our imaging survey, noting that this only $84 \%$ of $r_{\mathrm{vir}}$ is covered by our dataset. The faint end slope of an error weighted Schechter function fit reveals $\alpha=-1.32 \pm 0.05$. A similar fit to the luminosity function within the circular region fully covered by our data $\left(0.74 r_{\text {vir }}\right.$, see Fig. 9$)$, yields a marginally steeper slope $\alpha=-1.49 \pm 0.13$.

There is a hint of an upturn in the LF fainter than $M_{R}=$ $-12 \mathrm{mag}$, but completeness correction starts to play an important role in this magnitude range, such that we do not discuss it in more detail. 


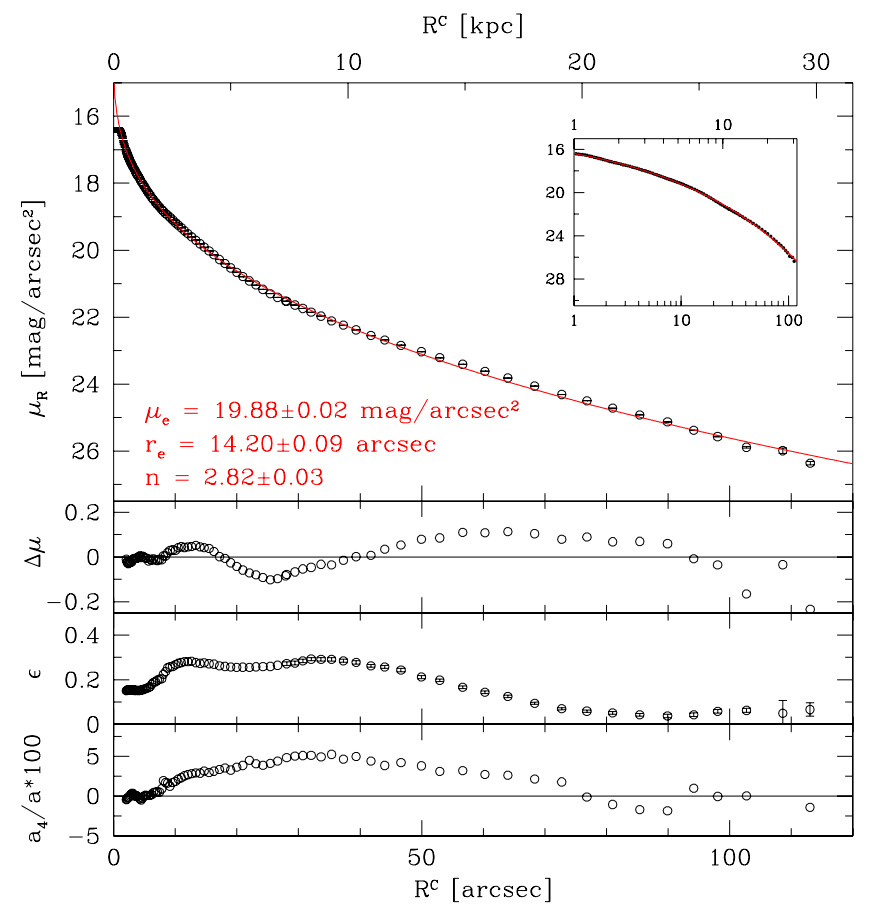

Fig. 13. Radial profiles of the BGG NGC 6482. Top panel: SB profile, including a plot of logarithmic radial scale in the inset. In both cases the red line represents the single Sérsic fit, the fitting results are provided too. The second panel displays the deviation of the data from the Sérsic fit. The third panel shows the ellipticity with respect to radial distance and the bottom panel illustrates the $a_{4} / a$ parameter that describes the deviation of the isophote from a perfect ellipse.

\subsection{The Brightest Group Galaxy NGC 6482}

The radial SB profile of the BGG (see Fig. 13) is well described by a single Sérsic fit as the deviation of the photometric data to the fit (second panel) within the inner 100 arcsec remains at values smaller than $\Delta \mu= \pm 0.1 \mathrm{mag} / \operatorname{arcsec}^{2}$. We fit the light profile with respect to the circularized radius and exclude the inner 3 arcsec (the inner 2 arcsec are obviously affected by saturation) from the fit. Its Sérsic $n=2.82 \pm 0.03-$ the light concentration parameter - is rather small for such a giant elliptical galaxy, given that a Sérsic $n=4$ represents the typical de Vaucouleurs profile. In particular it is smaller than reported by Alamo-Martínez et al. (2012) who find values of $~ 3.9$ in $g$ and $z$-band. However, smaller values of $n$ for fossil group central galaxies were already reported by the FOGO collaboration (Aguerri et al. 2011; Méndez-Abreu et al. 2012), who obtain a mean Sérsic index of $n \sim 3$ for a sample of 21 FGs.

The inner isophotes $\left(10^{\prime \prime} \lesssim R^{\mathrm{c}} \lesssim 50^{\prime \prime}\right)$ of NGC 6482 exhibit a moderately large ellipticity $(\epsilon \sim 0.3)$, and turn into almost spherical isophotes in the outskirts $\left(R^{\mathrm{c}}>70^{\prime \prime}\right)$. The elevated overall ellipticity is accompanied by a disky shape in the inner galaxy part (lower panel in Fig. 13). This is seen from the $a_{4} / a$ parameter, for which positive values denote disky isophotes (Bender 1988).

\subsection{A disrupted galaxy around MRK 895}

In one particular case we observe a galaxy being disrupted by the confirmed group member galaxy MRK 895. This is an edge-on S0 galaxy as shown in Fig. 14. For the disrupted galaxy we can only provide SExtractor-based photometry. We tuned SExtractor to detect the whole debris. The obtained flux represents only a lower limit because we have masked point sources, as well as the hosting S0 galaxy. This fact is denoted by the arrow on this galaxy in the CMD (Fig. 7).

We masked the tidal debris for the ellipse investigation of the S0 galaxy in the outer regions, but did not mask it where the debris crosses the bright isophotes along the disk of the galaxy. Thus, the isophotes might be affected by light from the tidal debris. But this effect should not be significant for the total brightness of the S0 galaxy since the galaxy itself is very bright (see light profile in the right panel of Fig. 14).

The projected diameter of the debris is $\sim 35 \mathrm{kpc}$. We investigated the $B-R$ colors of both the debris and the overdensity close to the northwest corner of MRK 0895 . While the overdensity shows $B-R \sim 1.4 \mathrm{mag}$, the rest of the debris shows $B-R$ colors of $\sim 1.2 \mathrm{mag}$. The S0 galaxy itself has $B-R \sim 1.69 \mathrm{mag}$ as shown in Fig. 7 (the third brightest galaxy is the host). As a result, the debris is on average $0.3 \mathrm{mag}$ bluer than its host. The disrupted galaxy is obviously not on the red sequence. The progenitor could have been a relatively luminous late-type and/or metal-poor system, since the flux of the whole debris adds to $M_{R}=-17.7 \mathrm{mag}$.

\section{Discussion and conclusions}

\subsection{Brightest group galaxy}

We find $B-R=1.73$ for the BGG NGC 6482, a color typical of metal-rich old stellar populations. Such a red color was also found for NGC 6482 by Alamo-Martínez et al. (2012) $\left(g-z=1.85\right.$ at $\left.r_{50}\right)$. Another finding of our study is the relatively low Sérsic index of $n \sim 2.82$ for NGC 6482, in agreement with the studies of the FOGO group, who find Sérsic indices around $n \sim 3$ for FGs (Aguerri et al. 2011; Méndez-Abreu et al. 2012). They argue based on Hopkins et al. (2009) and Kormendy et al. (2009) that low Sérsic indices for giant ellipticals $(n \sim 2.5)$ are tracers for dissipational mergers. Bender (1988) and Khochfar \& Burkert (2005) find that disky isophotes of giant elliptical galaxies are the result of wet, gas-rich mergers, i.e., mergers with participation of spiral or irregular galaxies. We clearly see disky isophotes in the BGG $\left(a_{4} / a \sim 0.05\right)$ and Alamo-Martínez et al. (2012) identify a dust lane in their HST images of NGC 6482 favoring the merger scenario of our FG as originally claimed by Ponman et al. (1994). But that does not necessarily disfavor the "failed group" scenario of Mulchaey \& Zabludoff (1999). Dekel et al. (2009) suggested that inflows of cold streams might have been the major formation scenario in the early Universe. A rotational disk would have stayed intact while giant star-forming clumps merged into the center to form a massive spheroid. These star-forming clumps - one could call them galaxies - could leave an imprint on the BGG's morphology, such as disky isophotes or dust lanes. Oser et al. (2010) show in simulations that the majority of stars of an intermediate mass central galaxy (like our NGC 6482) have been formed ex-situ in clumps, thus they have been accreted. In this sense, a massive dark matter halo accompanied by cold gas streams would reflect the "failed group" scenario but should also show merger signatures. A difference in both formation scenarios would then become washed-out.

\subsection{Photometric scaling relations}

We adopt the $\mu$-mag relation of Misgeld et al. (2009) to simulate galaxies in order to discover dwarf galaxies in the NGC 6482 group. These relations agree well with the sample properties of the recovered dwarfs as seen in Fig. 11. Disregarding disky 

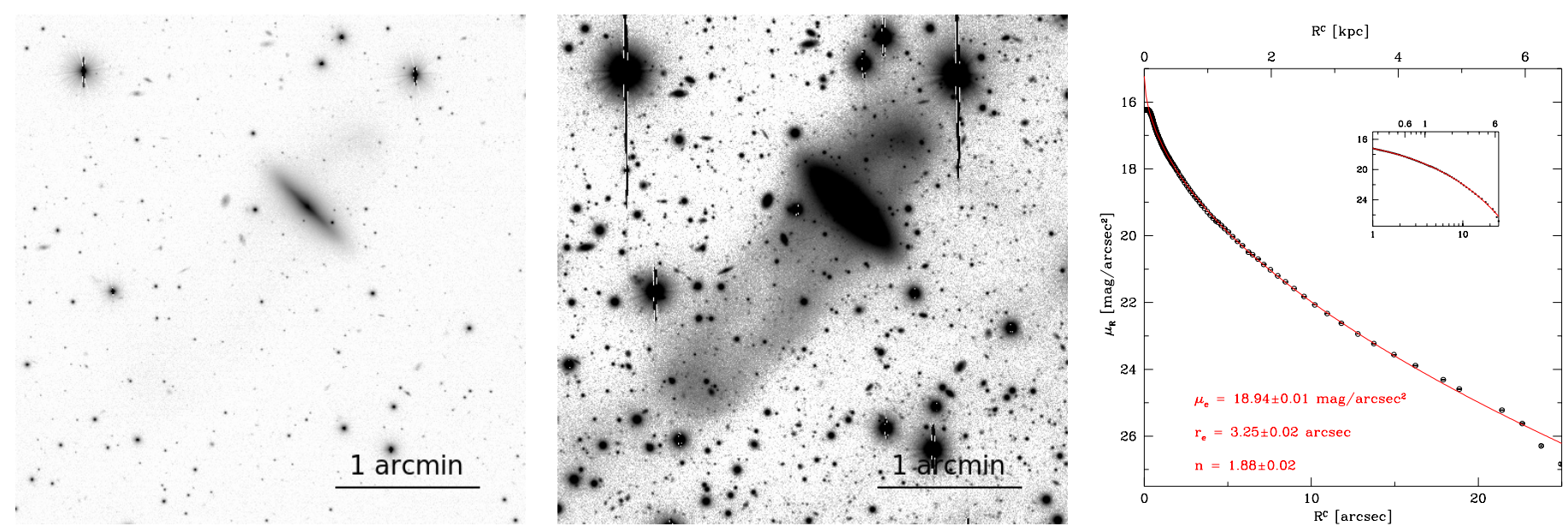

Fig. 14. Detection of a galaxy being disrupted. Left: S0 galaxy MRK 895 by which the dwarf galaxy is being disrupted (north up, east left). Center: image with different contrast settings to illustrate the tidal debris. Right: light profile of the S0 galaxy. The red line visualizes the best Sérsic model to the data.

galaxies (i.e., spirals and S0s), most galaxies in the sample follow the same photometric scaling relations, indicating that they truly belong to the NGC 6482 group. However, at the faint end of the distribution background contamination should play an important role. We accounted for that by applying a certain color range for group members, following the CMR of the bright galaxies.

In general, the photometric scaling relations are very similar to those found in galaxy clusters. This includes the distribution in CM space (see Fig. 7) where both the red and the blue sequence are distinctly defined. Our best fit of the CMR to the RS has a slope of $-0.029 \pm 0.008$, comparable to similar slope determinations in $B-R / R$ in some galaxy clusters $(-0.045 \pm 0.028$ for Coma; Adami et al. 2006; $-0.055 \pm 0.009$ for Perseus; Conselice et al. 2002), possibly somewhat to the shallow side.

\subsection{Luminosity function}

Little is known about the faint satellite systems of FGs. The deepest observational study of a FG in the literature reaches $M_{g}=-16 \mathrm{mag}$ (Mendes de Oliveira et al. 2006) - that is four magnitudes fainter than the group's characteristic magnitude $\left(M^{*}+4 \mathrm{mag}\right)$. Therefore no solid constraints on the dwarf galaxy regime can be drawn from these studies. Particularly the faint end slope of the LF has been poorly constrained up to now by other FG studies, and varies strongly from -0.6 to -1.6 (Cypriano et al. 2006; Mendes de Oliveira et al. 2006, 2009; Proctor et al. 2011; Eigenthaler \& Zeilinger 2012).

Our study is, to our knowledge, the first one to deeply probe into the dwarf galaxy regime of a fossil group, extending the available literature studies by 6-7 mag in total luminosity. Our investigation reveals dwarf galaxies as faint as $M_{R}=-10.5$ mag. We find a faint end slope of $\alpha=-1.32 \pm 0.05$, fully within the range of values typically found in cluster environments (Table 4), such as Coma or more unevolved clusters like Virgo and Hercules. The faint end slope of a composite LF, averaged over 60 nearby galaxy clusters, reveals a similar value of $\alpha=-1.28$ (De Propris et al. 2003). Previous studies have suggested that indeed the faint end slope of the LF is independent of environment: Trentham \& Tully (2002) show such an almost invariable faint end slope in their study of five different environments with varying galaxy density and morphological content.
Table 4. Luminosity function faint end slopes in literature.

\begin{tabular}{lccl}
\hline \hline Group/cluster & $\alpha$ & Band & Reference \\
\hline Virgo & -1.30 & $B$ & Sandage et al. (1985) \\
Coma & -1.41 & $R$ & Secker et al. (1997) \\
Hydra I & -1.13 & $V$ & Misgeld et al. (2008) \\
Centaurus & -1.14 & $V$ & Misgeld et al. (2009) \\
Hercules & -1.29 & $V$ & Sánchez-Janssen et al. (2005) \\
Fornax & -1.1 & $V$ & Hilker et al. (2003) \\
Perseus & -1.26 & $B$ & Penny \& Conselice (2008) \\
\hline Diff. env. $^{a}$ & -1.19 & $R$ & Trentham \& Tully (2002) \\
\hline 2dF & -1.28 & $b_{J}$ & De Propris et al. (2003) \\
\hline NGC 6482 & -1.32 & $R$ & this study \\
\hline
\end{tabular}

Notes. Faint end slopes $\alpha$ were derived by a single Schechter function fit. ${ }^{(a)}$ Composite LF (linear fit) of five different environments with varying galaxy density.

They find a composite faint end slope of $\alpha=-1.19$ for their entire sample. The result of our study, the first deep one in a fossil group, is consistent with the average slope found in a range of environments, and lends further credence to the notion that the faint end slope of the galaxy luminosity function depends only very little on environment.

Another useful parameter for describing the LF shape is the dwarf-to-giant ratio (Phillipps et al. 1998; Sánchez-Janssen et al. 2008). We compared our data with the study of Trentham $\&$ Tully (2002), adopting their definition of the $d / g$ ratio as $d / g=N\left(-17<M_{R}<-11\right) / N\left(M_{R}<-17\right)$. By taking the completeness correction into account, we obtain $d / g=4.1 \pm 0.6$. The error acounts for color and magnitude uncertainties that could propagate into our group membership determination. The dwarf-to-giant ratio we find is consistent with the average $d / g=$ 3.2 ( $\mathrm{ms} \mathrm{1.2)}$ reported in Trentham \& Tully (2002), and in particular matches the values they find for virialized systems like NGC 1407 and the Virgo cluster.

We do not find any galaxy in the magnitude range of $-14<$ $M_{R}<-13 \mathrm{mag}$, best seen in Fig. 11. While this interesting feature of NGC 6482 might be due to the low number counts (Fig. 12), we note that a similar dip has been found by Hilker et al. (2003) in the Fornax cluster. As already noted by these authors, this magnitude range is the transition from $\mathrm{dE}$ to $\mathrm{dSph}$. 
Another two properties of dwarf galaxies are the fraction of early- to late-types and the fraction of nucleated dEs. Trentham \& Tully (2002) find evidence that dynamically more evolved systems have a higher fraction of $\mathrm{dE}$ as compared to dIrr (see also Mahdavi et al. 2005). The percentage of dwarfs in the range $-17<M_{R}<-11$ mag classified $\mathrm{dE}$ as opposed to dIrr is $89_{-3}^{+1}$ per cent, comparable to the fraction Trentham \& Tully (2002) that find in the central $200 \mathrm{kpc}$ of Virgo cluster. The errorbars arise from photometric errors. Among the dEs, 38\% are nucleated within the same magnitude range. This is comparable to the $40 \%$ of a combined nucleation rate of four groups in the Trentham \& Tully (2002) sample but only half the nucleation they find for the Virgo cluster (70\%).

We conclude that the NGC 6482 fossil group shows photometric properties consistent with those of regular galaxy clusters and groups, including a normal abundance of faint satellites. The potential 'missing satellite problem' in this fossil group is thus on a similar scale to those in other environments.

Acknowledgements. Special thanks are addressed to Mischa Schirmer for his support on THELI issues with the tricky Subaru data. We also wish to thank the referee, Jairo Méndez-Abreu, for useful comments for improving the quality of this manuscript. S.L. is supported by the ESO Studentship Program. S.L. and T.L. are supported within the framework of the Excellence Initiative by the German Research Foundation (DFG) through the Heidelberg Graduate School of Fundamental Physics (grant number GSC 129/1). This research made use of the NASA/IPAC Extragalactic Database (NED), which is operated by the Jet Propulsion Laboratory, California Institute of Technology, under contract with the National Aeronautics and Space Administration.

\section{References}

Adami, C., Picat, J. P., Savine, C., et al. 2006, A\&A, 451, 1159

Aguerri, J. A. L., Girardi, M., Boschin, W., et al. 2011, A\&A, 527, A143

Alamo-Martínez, K. A., West, M. J., Blakeslee, J. P., et al. 2012, A\&A, 546, A15

Bender, R. 1988, A\&A, 193, L7

Bertin, E., \& Arnouts, S. 1996, A\&AS, 117, 393

Böhringer, H., Voges, W., Huchra, J. P., et al. 2000, ApJS, 129, 435

Caon, N., Capaccioli, M., \& D’Onofrio, M. 1993, MNRAS, 265, 1013

Caon, N., Cairós, L. M., Aguerri, J. A. L., \& Muñoz Tuñón, C. 2005, ApJS, 157, 218

Conselice, C. J., Gallagher, III, J. S., \& Wyse, R. F. G. 2002, AJ, 123, 2246

Cui, W., Springel, V., Yang, X., De Lucia, G., \& Borgani, S. 2011, MNRAS, 416, 2997

Cypriano, E. S., Mendes de Oliveira, C. L., \& Sodré, Jr., L. 2006, AJ, 132, 514 Dariush, A., Khosroshahi, H. G., Ponman, T. J., et al. 2007, MNRAS, 382, 433 Dariush, A. A., Raychaudhury, S., Ponman, T. J., et al. 2010, MNRAS, 405, 1873 De Propris, R., Colless, M., Driver, S. P., et al. 2003, MNRAS, 342, 725 Dekel, A., Birnboim, Y., Engel, G., et al. 2009, Nature, 457, 451

Díaz-Giménez, E., Muriel, H., \& Mendes de Oliveira, C. 2008, A\&A, 490, 965 D’Onghia, E., \& Lake, G. 2004, ApJ, 612, 628
D’Onghia, E., Sommer-Larsen, J., Romeo, A. D., et al. 2005, ApJ, 630, L109 Eigenthaler, P., \& Zeilinger, W. W. 2012, A\&A, 540, A134

Epanechnikov, V. 1969, Theory of Probability and its Applications, 14, 156

Erben, T., Schirmer, M., Dietrich, J. P., et al. 2005, Astron. Nachr., 326, 432

Hilker, M., Mieske, S., \& Infante, L. 2003, A\&A, 397, L9

Hopkins, P. F., Cox, T. J., Dutta, S. N., et al. 2009, ApJS, 181, 135

Janz, J., Laurikainen, E., Lisker, T., et al. 2012, ApJ, 745, L24

Jedrzejewski, R. I. 1987, MNRAS, 226, 747

Jones, L. R., Ponman, T. J., \& Forbes, D. A. 2000, MNRAS, 312, 139

Jones, L. R., Ponman, T. J., Horton, A., et al. 2003, MNRAS, 343, 627

Khochfar, S., \& Burkert, A. 2005, MNRAS, 359, 1379

Khosroshahi, H. G., Jones, L. R., \& Ponman, T. J. 2004, MNRAS, 349, 1240

Khosroshahi, H. G., Ponman, T. J., \& Jones, L. R. 2006, MNRAS, 372, L68

Kormendy, J. 1977, ApJ, 218, 333

Kormendy, J., \& Bender, R. 2012, ApJS, 198, 2

Kormendy, J., Fisher, D. B., Cornell, M. E., \& Bender, R. 2009, ApJS, 182, 216

Landolt, A. U. 1992, AJ, 104, 340

Lieder, S., Lisker, T., Hilker, M., Misgeld, I., \& Durrell, P. 2012, A\&A, 538, A69

Mahdavi, A., Trentham, N., \& Tully, R. B. 2005, AJ, 130, 1502

Mendes de Oliveira, C. L., Cypriano, E. S., Dupke, R. A., \& Sodré, Jr., L. 2009, AJ, 138, 502

Mendes de Oliveira, C. L., Cypriano, E. S., \& Sodré, Jr., L. 2006, AJ, 131, 158

Méndez-Abreu, J., Aguerri, J. A. L., Barrena, R., et al. 2012, A\&A, 537, A25

Milosavljevi, M., Miller, C. J., Furlanetto, S. R., \& Cooray, A. 2006, ApJ, 637, L9

Misgeld, I., \& Hilker, M. 2011, MNRAS, 414, 3699

Misgeld, I., Mieske, S., \& Hilker, M. 2008, A\&A, 486, 697

Misgeld, I., Hilker, M., \& Mieske, S. 2009, A\&A, 496, 683

Miyazaki, S., Komiyama, Y., Sekiguchi, M., et al. 2002, PASJ, 54, 833

Mulchaey, J. S., \& Zabludoff, A. I. 1999, ApJ, 514, 133

Oser, L., Ostriker, J. P., Naab, T., Johansson, P. H., \& Burkert, A. 2010, ApJ, 725,2312

Penny, S. J., \& Conselice, C. J. 2008, MNRAS, 383, 247

Phillipps, S., Driver, S. P., Couch, W. J., \& Smith, R. M. 1998, ApJ, 498, L119

Ponman, T. J., Allan, D. J., Jones, L. R., et al. 1994, Nature, 369, 462

Proctor, R. N., de Oliveira, C. M., Dupke, R., et al. 2011, MNRAS, 418, 2054

Sánchez-Janssen, R., Aguerri, J. A. L., \& Muñoz Tuñón, C. 2008, ApJ, 679, L77

Sánchez-Janssen, R., Iglesias-Páramo, J., Muñoz Tuñón, C., Aguerri, J. A. L., \& Vílchez, J. M. 2005, A\&A, 434, 521

Sandage, A., \& Binggeli, B. 1984, AJ, 89, 919

Sandage, A., Binggeli, B., \& Tammann, G. A. 1985, AJ, 90, 1759

Sanderson, A. J. R., Ponman, T. J., Finoguenov, A., Lloyd-Davies, E. J., \& Markevitch, M. 2003, MNRAS, 340, 989

Schlegel, D. J., Finkbeiner, D. P., \& Davis, M. 1998, ApJ, 500, 525

Secker, J., Harris, W. E., \& Plummer, J. D. 1997, PASP, 109, 1377

Sérsic, J. L. 1968, Atlas de galaxias australes (Cordoba, Argentina: Observatorio Astronomico)

Smith, R. J., Lucey, J. R., Hudson, M. J., Schlegel, D. J., \& Davies, R. L. 2000, MNRAS, 313, 469

Trentham, N., \& Tully, R. B. 2002, MNRAS, 335, 712

von Benda-Beckmann, A. M., D’Onghia, E., Gottlber, S., et al. 2008, MNRAS, 386,2345

Worthey, G. 1994, ApJS, 95, 107

Zabludoff, A. I., \& Mulchaey, J. S. 1998, ApJ, 496, 39

Zwicky, F., Herzog, E., \& Wild, P. 1963, Catalogue of galaxies and of clusters of galaxies (Pasadena: California Institute of Technology), 2 


\section{Appendix A: Tables}

Table A.1. Properties of all galaxies considered as group members in this study.

\begin{tabular}{|c|c|c|c|c|c|c|c|c|c|}
\hline ID & $\begin{array}{c}\alpha(\mathrm{J} 2000) \\
{[\mathrm{deg}]}\end{array}$ & $\begin{array}{c}\delta(\mathrm{J} 2000) \\
{[\mathrm{deg}]}\end{array}$ & $\begin{array}{c}M_{R} \\
{[\mathrm{mag}]}\end{array}$ & $\begin{array}{c}M_{B} \\
{[\mathrm{mag}]}\end{array}$ & $\begin{array}{l}B-R \\
{[\mathrm{mag}]}\end{array}$ & $\begin{array}{c}\mu_{\mathrm{e}} \\
{\left[\mathrm{mag} / \operatorname{arcsec}^{2}\right]}\end{array}$ & $\begin{array}{c}r_{\mathrm{e}} \\
{[\mathrm{kpc}]}\end{array}$ & $n$ & Type \\
\hline $1^{a}$ & 267.95346 & 23.07192 & $-22.73 \pm 0.02$ & $-21.09 \pm 0.02$ & $1.73 \pm 0.02^{c}$ & $19.88 \pm 0.02$ & $3.73 \pm 0.13$ & $2.82 \pm 0.03$ & $\mathrm{E}$ \\
\hline $2^{a}$ & 268.22717 & 23.21117 & $-20.54 \pm 0.04$ & $-18.92 \pm 0.07$ & $1.72 \pm 0.07$ & $21.01 \pm 0.01$ & $2.42 \pm 0.13$ & $1.05 \pm 0.01$ & $\mathrm{Sc}$ \\
\hline $3^{a}$ & 267.69073 & 23.13864 & $-20.26 \pm 0.02$ & $-18.64 \pm 0.03$ & $1.69 \pm 0.02$ & $18.94 \pm 0.01$ & $0.85 \pm 0.13$ & $1.88 \pm 0.02$ & S0 \\
\hline $4^{a}$ & 268.20694 & 23.01428 & $-19.69 \pm 0.04$ & $-18.53 \pm 0.05$ & $1.19 \pm 0.06$ & $21.84 \pm 0.02$ & $2.88 \pm 0.13$ & $1.07 \pm 0.03$ & $\mathrm{SBc}$ \\
\hline 5 & 268.09314 & 22.87197 & $-19.15 \pm 0.09$ & $-17.97 \pm 0.09$ & $1.19 \pm 0.07$ & $23.51 \pm 0.03$ & $4.99 \pm 0.14$ & $0.60 \pm 0.03$ & $\mathrm{SBc}$ \\
\hline 6 & 267.90015 & 23.07572 & $-18.38 \pm 0.01$ & $-16.75 \pm 0.03$ & $1.60 \pm 0.01$ & $21.71 \pm 0.02$ & $1.36 \pm 0.13$ & $1.38 \pm 0.03$ & $\mathrm{~S}($ lens $) 0$ \\
\hline 7 & 267.68355 & 23.14623 & $-17.70_{+0.01}^{-0.5}$ & $-16.37_{+0.02}^{-0.5}$ & $1.33 \pm 0.13^{b}$ & - & - & - & disrupted \\
\hline 8 & 267.74030 & 22.90452 & $-17.66 \pm 0.02$ & $-16.25 \pm 0.02$ & $1.53 \pm 0.02$ & $22.44 \pm 0.01$ & $1.42 \pm 0.13$ & $1.42 \pm 0.01$ & $\mathrm{dE}, \mathrm{N}$ \\
\hline 9 & 267.78040 & 23.14328 & $-17.23 \pm 0.05$ & $-16.17 \pm 0.05$ & $1.07 \pm 0.05$ & $23.05 \pm 0.01$ & $1.59 \pm 0.13$ & $1.12 \pm 0.02$ & dIrr \\
\hline 10 & 267.84186 & 23.06776 & $-16.51 \pm 0.02$ & $-15.08 \pm 0.04$ & $1.55 \pm 0.01$ & $23.09 \pm 0.01$ & $1.13 \pm 0.13$ & $1.34 \pm 0.01$ & $\mathrm{dE}, \mathrm{N}$ \\
\hline 11 & 268.22913 & 23.11805 & $-16.35 \pm 0.03$ & $-14.96 \pm 0.06$ & $1.52 \pm 0.02$ & $23.81 \pm 0.01$ & $1.22 \pm 0.13$ & $1.09 \pm 0.01$ & $\mathrm{dE}, \mathrm{N}$ \\
\hline 12 & 267.85382 & 23.06654 & $-15.90 \pm 0.04$ & $-14.53 \pm 0.05$ & $1.42 \pm 0.03$ & $24.20 \pm 0.01$ & $1.62 \pm 0.13$ & $0.74 \pm 0.01$ & $\mathrm{dE}$ \\
\hline 13 & 267.76700 & 22.85475 & $-15.76 \pm 0.05$ & $-14.38 \pm 0.08$ & $1.47 \pm 0.02$ & $23.68 \pm 0.01$ & $1.01 \pm 0.13$ & $1.20 \pm 0.02$ & $\mathrm{dE}, \mathrm{N}$ \\
\hline 14 & 267.91089 & 23.12349 & $-15.67 \pm 0.04$ & $-14.35 \pm 0.06$ & $1.41 \pm 0.03$ & $23.66 \pm 0.02$ & $1.02 \pm 0.13$ & $1.54 \pm 0.02$ & $\mathrm{dE}, \mathrm{N}$ \\
\hline 15 & 267.76242 & 23.04759 & $-15.30 \pm 0.05$ & $-14.17 \pm 0.06$ & $1.11 \pm 0.08$ & $23.72 \pm 0.02$ & $0.73 \pm 0.13$ & $0.55 \pm 0.01$ & dIrr \\
\hline 16 & 267.66498 & 23.25852 & $-15.08 \pm 0.07$ & $-13.90 \pm 0.08$ & $1.19 \pm 0.03$ & $23.90 \pm 0.02$ & $0.96 \pm 0.13$ & $0.85 \pm 0.02$ & dIrr? \\
\hline 17 & 267.81354 & 23.30462 & $-14.64 \pm 0.03$ & $-13.48 \pm 0.05$ & $1.14 \pm 0.03$ & $23.95 \pm 0.02$ & $0.71 \pm 0.13$ & $1.08 \pm 0.04$ & dIrr \\
\hline 18 & 267.68402 & 22.87308 & $-14.61 \pm 0.07$ & $-13.09 \pm 0.09$ & $1.55 \pm 0.02$ & $24.82 \pm 0.02$ & $1.16 \pm 0.13$ & $0.87 \pm 0.01$ & $\mathrm{dE}$ \\
\hline 19 & 268.04355 & 22.84158 & $-14.22 \pm 0.05$ & $-12.83 \pm 0.12$ & $1.48 \pm 0.04$ & $24.01 \pm 0.01$ & $0.63 \pm 0.13$ & $0.99 \pm 0.01$ & $\mathrm{dE}, \mathrm{N}$ \\
\hline 20 & 267.75461 & 23.08187 & $-14.21 \pm 0.08$ & $-12.92 \pm 0.25$ & $2.48_{-1.13}^{+0.12}$ & $25.91 \pm 0.09$ & $2.37 \pm 0.20$ & $1.32 \pm 0.06$ & $\mathrm{dE}$ \\
\hline 21 & 267.69376 & 22.86386 & $-14.19 \pm 0.07$ & $-12.70 \pm 0.08$ & $1.57 \pm 0.03$ & $24.22 \pm 0.01$ & $0.68 \pm 0.13$ & $0.71 \pm 0.01$ & $\mathrm{dE}, \mathrm{N}$ \\
\hline 22 & 267.93338 & 23.09780 & $-14.11 \pm 0.15$ & $-12.52 \pm 0.35$ & $1.74 \pm 0.13$ & $25.84 \pm 0.14$ & $2.28 \pm 0.27$ & $1.11 \pm 0.09$ & $\mathrm{dE}, \mathrm{N}$ \\
\hline 23 & 267.84854 & 23.17222 & $-12.95 \pm 0.21$ & $-11.61 \pm 0.19$ & $1.38 \pm 0.09$ & $25.47 \pm 0.02$ & $0.73 \pm 0.13$ & $0.66 \pm 0.02$ & $\mathrm{dE}$ \\
\hline 24 & 267.72772 & 23.02693 & $-12.91 \pm 0.10$ & $-11.60 \pm 0.14$ & $1.41 \pm 0.05$ & $24.16 \pm 0.01$ & $0.41 \pm 0.13$ & $0.60 \pm 0.02$ & $\mathrm{dE}$ \\
\hline 25 & 267.82730 & 23.01918 & $-12.87 \pm 0.05$ & $-11.69 \pm 0.10$ & $1.43 \pm 0.04$ & $24.56 \pm 0.02$ & $0.49 \pm 0.13$ & $1.03 \pm 0.02$ & $\mathrm{dE}$ \\
\hline 26 & 267.89713 & 23.13568 & $-12.70 \pm 0.08$ & $-11.37 \pm 0.15$ & $1.48 \pm 0.07$ & $25.19 \pm 0.02$ & $0.57 \pm 0.13$ & $0.60 \pm 0.02$ & $\mathrm{dE}, \mathrm{N}$ \\
\hline 27 & 268.15881 & 23.30970 & $-12.64 \pm 0.09$ & $-11.73 \pm 0.12$ & $0.78 \pm 0.07$ & $24.64 \pm 0.01$ & $0.43 \pm 0.13$ & $0.71 \pm 0.02$ & $\mathrm{dE}$ \\
\hline 28 & 267.75369 & 22.86655 & $-12.54 \pm 0.06$ & $-11.17 \pm 0.13$ & $1.50 \pm 0.04$ & $25.18 \pm 0.02$ & $0.53 \pm 0.13$ & $0.78 \pm 0.02$ & $\mathrm{dE}$ \\
\hline 29 & 267.97821 & 22.92692 & $-12.53 \pm 0.18$ & $-11.21 \pm 0.32$ & $1.31 \pm 0.11$ & $27.14 \pm 0.08$ & $0.88 \pm 0.14$ & $0.88 \pm 0.06$ & $\mathrm{dE}$ \\
\hline 30 & 267.80301 & 23.05564 & $-12.34 \pm 0.07$ & $-11.12 \pm 0.22$ & $1.43 \pm 0.05$ & $25.53 \pm 0.04$ & $0.54 \pm 0.13$ & $0.86 \pm 0.04$ & $\mathrm{dE}, \mathrm{N}$ \\
\hline 31 & 268.06989 & 23.01075 & $-12.33 \pm 0.06$ & $-10.94 \pm 0.17$ & $1.52 \pm 0.06$ & $25.66 \pm 0.04$ & $0.67 \pm 0.13$ & $0.81 \pm 0.03$ & $\mathrm{dE}, \mathrm{N}$ \\
\hline 32 & 267.97345 & 22.99362 & $-12.16 \pm 0.04$ & $-10.83 \pm 0.10$ & $1.37 \pm 0.03$ & $24.81 \pm 0.02$ & $0.39 \pm 0.13$ & $0.84 \pm 0.03$ & $\mathrm{dE}$ \\
\hline 33 & 267.83710 & 23.06381 & $-11.94 \pm 0.04$ & $-10.64 \pm 0.10$ & $1.46 \pm 0.03$ & $24.57 \pm 0.01$ & $0.32 \pm 0.13$ & $0.63 \pm 0.01$ & $\mathrm{dE}$ \\
\hline 34 & 268.01102 & 23.16201 & $-11.57 \pm 0.09$ & $-10.63 \pm 0.08$ & $1.12 \pm 0.05$ & $24.30 \pm 0.02$ & $0.23 \pm 0.13$ & $0.69 \pm 0.04$ & $\mathrm{dE}$ \\
\hline 35 & 267.69958 & 23.06408 & $-11.55 \pm 0.08$ & $-10.36 \pm 0.17$ & $1.36 \pm 0.08$ & $25.97 \pm 0.07$ & $0.50 \pm 0.13$ & $0.59 \pm 0.05$ & $\mathrm{dE}$ \\
\hline 36 & 267.87686 & 23.19275 & $-11.44 \pm 0.07$ & $-10.06 \pm 0.12$ & $1.54 \pm 0.11$ & $24.71 \pm 0.01$ & $0.29 \pm 0.13$ & $0.34 \pm 0.01$ & $\mathrm{dE}$ \\
\hline 37 & 267.92081 & 23.14277 & $-11.29 \pm 0.09$ & $-10.20 \pm 0.24$ & $1.34 \pm 0.07$ & $26.14 \pm 0.13$ & $0.55 \pm 0.14$ & $0.64 \pm 0.09$ & $\mathrm{dE}$ \\
\hline 38 & 268.07532 & 23.29704 & $-11.03 \pm 0.14$ & $-10.21 \pm 0.15$ & $0.96 \pm 0.12$ & $25.51 \pm 0.03$ & $0.33 \pm 0.13$ & $0.42 \pm 0.02$ & $\mathrm{dE}$ \\
\hline 39 & 268.19034 & 23.20517 & $-10.92 \pm 0.12$ & $-9.80 \pm 0.44$ & $1.23 \pm 0.10$ & $26.46 \pm 0.37$ & $0.47 \pm 0.17$ & $1.01 \pm 0.33$ & $\mathrm{dE}, \mathrm{N}$ \\
\hline 40 & 268.02652 & 22.90529 & $-10.77 \pm 0.18$ & $-9.20 \pm 0.32$ & $1.49 \pm 0.15$ & $26.53 \pm 0.34$ & $0.52 \pm 0.20$ & $0.72 \pm 0.22$ & $\mathrm{dE}$ \\
\hline 41 & 267.84235 & 22.85096 & $-10.76 \pm 0.09$ & $-9.47 \pm 0.11$ & $1.52 \pm 0.10$ & $24.92 \pm 0.02$ & $0.24 \pm 0.13$ & $0.36 \pm 0.02$ & $\mathrm{dE}$ \\
\hline 42 & 267.67389 & 23.22414 & $-10.75 \pm 0.14$ & $-8.78 \pm 0.25$ & $1.54 \pm 0.18$ & $26.92 \pm 0.21$ & $0.40 \pm 0.16$ & $0.34 \pm 0.11$ & $\mathrm{dE}$ \\
\hline 43 & 267.97455 & 23.11946 & $-10.71 \pm 0.14$ & $-9.29 \pm 0.33$ & $1.46 \pm 0.21$ & $27.79 \pm 0.27$ & $0.29 \pm 0.15$ & $0.67 \pm 0.16$ & $\mathrm{dE}$ \\
\hline 44 & 267.67850 & 22.96362 & $-10.67 \pm 0.09$ & $-9.76 \pm 0.16$ & $1.07 \pm 0.07$ & $25.19 \pm 0.01$ & $0.23 \pm 0.13$ & $0.29 \pm 0.01$ & $\mathrm{dE}$ \\
\hline 45 & 267.85178 & 23.01907 & $-10.61 \pm 0.17$ & $-9.83 \pm 0.22$ & $0.95 \pm 0.12$ & $27.06 \pm 0.14$ & $0.48 \pm 0.14$ & $0.62 \pm 0.08$ & $\mathrm{dE}$ \\
\hline 46 & 268.05835 & 22.93668 & $-10.59 \pm 0.10$ & $-9.36 \pm 0.21$ & $1.53 \pm 0.09$ & $25.47 \pm 0.04$ & $0.27 \pm 0.13$ & $0.52 \pm 0.05$ & $\mathrm{dE}$ \\
\hline 47 & 268.10507 & 23.04556 & $-10.55 \pm 0.20$ & $-9.52 \pm 0.25$ & $1.31 \pm 0.12$ & $25.64 \pm 0.04$ & $0.31 \pm 0.13$ & $0.47 \pm 0.04$ & $\mathrm{dE}$ \\
\hline 48 & 268.06409 & 22.86462 & $-10.53 \pm 0.30$ & $-9.84 \pm 0.29$ & $1.08 \pm 0.16$ & $25.94 \pm 0.08$ & $0.28 \pm 0.13$ & $0.76 \pm 0.08$ & $\mathrm{dE}$ \\
\hline
\end{tabular}

Notes. ID: galaxy identification number in our catalog sorted by $M_{R} . \alpha(\mathrm{J} 2000)$ : Right ascension. $\delta$ (J2000): Declination. $M_{R}$ : absolute $R$-band magnitude (adopted $m-M=33.71 \mathrm{mag}$ ), errorbars do not include photometric calibration uncertainty of $\sigma_{R}=0.04$ mag. $M_{B}$ : absolute $B$-band magnitude (adopted $m-M=33.71 \mathrm{mag}$ ); errorbars do not include photometric calibration uncertainty of $\sigma_{B}=0.12 \mathrm{mag}$. $B-R$ : integrated $B-R$ color (within half-light radius $r_{50}$ ). $\mu_{\mathrm{e}}$ : effective surface brightness in $R$-band from Sérsic fit in $R$-band applied to circularized isophotes. $r_{\mathrm{e}}$ : effective radius from Sérsic fit in $R$-band applied to circularized isophotes, errors include uncertainty introduced by seeing. $n$ : Sérsic index. Type: classification type (for member considered galaxies only; all early-type dwarf galaxies are labeled dE). ${ }^{(a)}$ Objects with NED-listed velocities, qualiyfing the galaxy as a group member. ${ }^{(b)}$ Color determined using SExtractor. ${ }^{(c)}$ Color determined at half-light radius. 